\title{
Exploring CP violation in the MSSM
}

\author{
Alexandre Arbey ${ }^{1,2,3}$, John Ellis ${ }^{3,4}$, Rohini M. Godbole ${ }^{5}$, Farvah Mahmoudi ${ }^{1,2,3,6, a}$ \\ ${ }^{1}$ Université de Lyon, Université Lyon 1, Centre de Recherche Astrophysique de Lyon, CNRS, UMR 5574, 69561 Saint-Genis Laval Cedex, France \\ ${ }^{2}$ Ecole Normale Supérieure de Lyon, Lyon, France \\ 3 Theory Division, CERN, 1211 Geneva 23, Switzerland \\ ${ }^{4}$ Theoretical Particle Physics and Cosmology Group, Department of Physics, King's College London, London WC2R 2LS, UK \\ ${ }_{6}^{5}$ Centre for High Energy Physics, Indian Institute of Science, Bangalore 560012, India \\ ${ }^{6}$ Institut Universitaire de France, 103 boulevard Saint-Michel, 75005 Paris, France
}

Received: 23 October 2014 / Accepted: 28 January 2015 / Published online: 21 February 2015

(C) The Author(s) 2015. This article is published with open access at Springerlink.com

\begin{abstract}
We explore the prospects for observing CP violation in the minimal supersymmetric extension of the Standard Model (MSSM) with six CP-violating parameters, three gaugino mass phases and three phases in trilinear soft supersymmetry-breaking parameters, using the $\mathrm{CP}$ super $\mathrm{H}$ code combined with a geometric approach to maximise CP-violating observables subject to the experimental upper bounds on electric dipole moments. We also implement CP-conserving constraints from Higgs physics, flavour physics and the upper limits on the cosmological dark matter density and spin-independent scattering. We study possible values of observables within the constrained MSSM (CMSSM), the non-universal Higgs model (NUHM), the CPX scenario and a variant of the phenomenological MSSM (pMSSM). We find values of the CP-violating asymmetry $A_{\mathrm{CP}}$ in $b \rightarrow s \gamma$ decay that may be as large as $3 \%$, so future measurements of $A_{\mathrm{CP}}$ may provide independent information about $\mathrm{CP}$ violation in the MSSM. We find that CP-violating MSSM contributions to the $B_{s}$ meson mass mixing term $\Delta M_{B_{s}}$ are in general below the present upper limit, which is dominated by theoretical uncertainties. If these could be reduced, $\Delta M_{B_{s}}$ could also provide an interesting and complementary constraint on the six CP-violating MSSM phases, enabling them all to be determined experimentally, in principle. We also find that CP violation in the $h_{2,3} \tau^{+} \tau^{-}$and $h_{2,3} \bar{t} t$ couplings can be quite large, and so may offer interesting prospects for future $p p, e^{+} e^{-}, \mu^{+} \mu^{-}$and $\gamma \gamma$ colliders.
\end{abstract}

\footnotetext{
a e-mail: mahmoudi@in2p3.fr
}

\section{Introduction}

The minimal supersymmetric extension of the Standard Model (MSSM) contains many possible sources of CP violation beyond the Kobayashi-Maskawa phase of the Standard Model and the strong CP phase. These additional sources of $\mathrm{CP}$ violation arise from the soft supersymmetry-breaking terms in the low-energy effective Lagrangian, and include phases in the gaugino masses, the trilinear scalar couplings and the sfermion mass matrices. However, the KobayashiMaskawa phase accounts very well for the CP-violating effects seen in the $K^{0}$ system and in $B$ meson decays, and no other violations of $\mathrm{CP}$ have been observed despite, for example, sensitive experimental searches for electric dipole moments (EDMs). Thus, it might be tempting to suggest that the extra MSSM sources of CP violation are absent. On the other hand, experimental upper limits still allow considerable scope for additional CP-violating effects in, for example, $B_{s}^{0}$ mixing, and some additional source of $\mathrm{CP}$ violation is needed to explain the cosmological baryon asymmetry, which might be due to these MSSM phases. For these reasons, there have been many studies of possible MSSM CP-violating effects in experimental observables, and powerful phenomenological tools have been developed for calculating these effects.

In view of the success of the Cabibbo-KobayashiMaskawa (CKM) model in describing flavour mixing and $\mathrm{CP}$ violation in the quark sector, it is often assumed that the strong CP phase is negligibly small for some reason, and that flavour and $\mathrm{CP}$ violation for squarks is generated by the CKM mixing in the quark sector, the hypothesis of minimal flavour violation (MFV). However, even in this case there remain several additional sources of $\mathrm{CP}$ violation in the MSSM, namely the phases in the gaugino masses and the trilinear couplings. One is thus led to consider the maximally $\mathrm{CP}$-violating, minimal flavour-violating (MCPMFV) model 
Table 1 The $95 \%$ CL upper limits on EDMs used as constraints in this study. The present experimental upper bound on the EDM of the muon, $1.9 \times 10^{-19}$ e.cm [10], provides only a very weak constraint that is not competitive with the other EDM constraints in the models discussed here

\begin{tabular}{lll}
\hline EDM & Upper limit (e.cm) & Reference \\
\hline Thallium & $1.3 \times 10^{-24}$ & {$[6]$} \\
Mercury & $3.5 \times 10^{-29}$ & {$[7]$} \\
Neutron & $4.7 \times 10^{-26}$ & {$[8]$} \\
Thorium monoxide & $1.1 \times 10^{-28}$ & {$[9]$} \\
\hline
\end{tabular}

that contains six CP-violating phases beyond the KobayashiMaskawa phase: three phases $\Phi_{1,2,3}$ in the masses of the $\mathrm{U}(1), \mathrm{SU}(2)$ and $\mathrm{SU}(3)$ gauginos, and three phases $\Phi_{A_{t, b, \tau}}$ in the trilinear soft supersymmetry-breaking couplings $A_{t, b, \tau}$ of the third-generation stop, sbottom and stau sfermions, respectively. ${ }^{1}$ In this study, we allow the six CP-violating phases to vary independently in all the scenarios considered. Predictions of the MCPMFV scenario for CP-violating observables such as the CP-violating asymmetry in $b \rightarrow s \gamma$ decay, $A_{\mathrm{CP}}$, the $\mathrm{CP}$-violating phase in $B_{s}$ mixing, $\phi_{s}$, and EDMs have been considered in [1,2], and possibilities for probing these $\mathrm{CP}$-violating phases through the polarisation of third-generation fermions, $t$ and $\tau$, produced in the decays of the corresponding sfermions have also been explored [3].

It might be thought that the MSSM phases $\Phi_{1,2,3, t, b, \tau}$ must necessarily be small, in view of the stringent upper limits on several EDMs shown in Table 1. However, this is not necessarily the case, since there are four main independent EDM constraints on what is, a priori, a 6-dimensional space of CP-violating MSSM phases, so there are in principle 'blind directions' corresponding to combinations of phases that do not 'see' the EDM constraints. In principle, individual phases could be large along these directions, as discussed in [4] for example, and could have significant effects on other CPviolating observables such as $A_{\mathrm{CP}}$ and $\phi_{s}{ }^{2}$

A brute force way to study this possibility would be to sample randomly the 6-dimensional space of CP-violating MSSM phases, but this is not the most efficient procedure to explore the possible magnitudes of CP-violating effects in the MSSM. If one wishes to generate a large sample of parameter sets that respect other phenomenological constraints such as those from the flavour, Higgs and dark matter sectors, one would prefer to optimise the search for MSSM scenarios with maximal CP violation. A geometric approach to this problem was proposed in [2] and used to analyse the impacts of

\footnotetext{
1 We assume that the strong $\mathrm{CP}$ phase is negligible, and also neglect the phases in the trilinear couplings of the sfermions in the first and second generations, which are much less important for phenomenology.

${ }^{2}$ We note in passing that there are also well-motivated supersymmetric models in which the phases are naturally small, so that the EDM bounds are not very constraining, see [5] for example.
}

three EDM constraints in certain specific benchmark MSSM scenarios.

In this paper we adapt and extend this geometric approach to study systematically the possible magnitudes of CPviolating effects in light of the updated EDM constraints shown in Table 1. The inclusion of a fourth EDM constraint requires a slight extension of the analysis based on three EDMs made in [2], as we discuss in Sect. 2. Also, the geometric approach was originally formulated as a linear expansion around the $\mathrm{CP}$-conserving limit, whereas we are interested in the largest possible values of the $\mathrm{CP}$-violating phases. Accordingly, here we extend the approach using an iterative procedure, finding an initial 'blind direction' as in [2], then choosing a $\mathrm{CP}$-violating point along that direction with nonzero phases, and then repeating the geometrical optimisation in a new linear approximation around this $\mathrm{CP}$-violating point, as also discussed in Sect. 2. In Sect. 3 we then apply the geometric approach to four variants of the MSSM, a best-fit scenario $[11,12]$ within the constrained MSSM (CMSSM) in which the soft supersymmetry-breaking parameters are constrained to be universal at the GUT scale (apart from the CP-violating phases), a generalisation of this model in which the soft supersymmetry-breaking contributions to the two Higgs doublet masses are allowed to vary independently (NUHM2), a version of the CPX scenario defined in [13] that is modified to be in agreement with the LHC results, and the phenomenological MSSM (pMSSM) [14], in which extrapolation to the GUT scale is ignored and universality is not imposed. ${ }^{3}$ In each case, in addition to the EDM constraints in Table 1, we also consider the relevant constraints from flavour physics, from the measured properties of the known Higgs boson and searches for other MSSM Higgs bosons, and upper limits on the cosmological density of dark matter and the direct detection of dark matter via scattering on nuclei.

We focus, in particular, on four possible signatures of MSSM CP violation: the possibility that there might be another neutral Higgs boson lighter than the one already discovered by ATLAS and CMS, the CP-violating asymmetry in $b \rightarrow s \gamma$ decay, $A_{\mathrm{CP}}$, and the non-Standard-Model contribution to the $B_{s}$ meson mixing parameter, $\Delta M_{B_{s}}$, and $\mathrm{CP}$-violating couplings of the heavier neutral Higgs bosons. We find that, although a neutral MSSM Higgs boson lighter than that discovered would be consistent with the EDM constraints, it is excluded by the available limits on other Higgs bosons, notably the absence of a light charged Higgs boson. Secondly, we find that values of $A_{\mathrm{CP}} \lesssim 3 \%$ are allowed by the EDMs and other constraints in some of the MSSM scenarios studied. This opens up the possibility that $A_{\mathrm{CP}}$ could be significantly larger than in the Standard Model, providing a signature of CP-violating MSSM. Conversely,

\footnotetext{
${ }^{3}$ Partial results in the case of CMSSM were presented in [15].
} 
if a non-zero value of $A_{\mathrm{CP}}$ were not to be found in future experiments, this could provide a constraint on $\mathrm{CP}$ violation in the MSSM that is independent of, and complementary to, those from EDMs. Thirdly, in the case of $\Delta M_{B_{s}}$, we find that it could also provide an independent constraint on the CP-violating MSSM if the theoretical uncertainties could be reduced, thereby enabling in principle a complete determination of all the phases for fixed values of the CP-conserving MSSM parameters. Fourthly, we also find that $\mathrm{CP}$ violation in the $h_{2,3} \tau^{+} \tau^{-}$and $h_{2,3} \bar{t} t$ couplings can be quite large, and may offer interesting prospects for future $p p, e^{+} e^{-}$and $\mu^{+} \mu^{-}$experiments.

\section{Method}

In this section we outline our approach to sampling the parameter spaces of MSSM scenarios while respecting the four EDM constraints in Table 1. Since the EDM constraints are quite strong, they effectively reduce the dimensionality of any MSSM scenario by four. The challenge is to sample efficiently this subspace of codimension four, so as to assess how large any other CP-violating observable may be. Moreover, the thorium monoxide EDM constraint on the electron EDM is now so strong that we have designed a new method to sample effectively the parameter space. We do this by adapting and extending the geometric approach proposed in [2]. In the first subsection we discuss how the approach may be modified to take into account four EDM constraints, in the following subsection we describe an extension of the analysis beyond the small-phase approximation, and in the third subsection we summarise our sampling algorithm.

\subsection{Geometric approach to maximizing a CP-violating observable with four EDM constraints}

Initially, we consider the four EDMs $E^{a, b, c, d}$ of Table 1 in the small-phase approximation, ${ }^{4}$ where

$E^{i} \simeq \boldsymbol{\Phi} \cdot \mathbf{E}^{i}$,

with $\boldsymbol{\Phi} \equiv \Phi_{\alpha}=\Phi_{1,2,3, t, b, \tau}$ and $\mathbf{E}^{i} \equiv \partial E^{i} / \partial \boldsymbol{\Phi}$ (i.e., $E_{\alpha}^{i} \equiv$ $\left.\partial E^{i} / \partial \Phi_{\alpha}\right)$. The $\boldsymbol{\Phi}$ subspace of codimension four is spanned by the following quadruple exterior product:

$A_{\alpha \beta \gamma \delta}=E_{[\alpha}^{a} E_{\beta}^{b} E_{\gamma}^{c} E_{\delta]}^{d}$

where the symbols [...] denote antisymmetrisation of the enclosed indices. This subspace is a 2-dimensional plane, as in the simple example in Section 2.1 of [2]. We now consider some CP-violating observable $O$ whose dependence on the phases $\Phi_{\alpha}$ is given in the small-phase approximation

\footnotetext{
${ }^{4}$ We use Latin indices $i, j, \ldots$ for the EDMs, and Greek indices $\alpha, \beta, \ldots$ for the $\mathrm{CP}$-violating phases.
}

by $\mathbf{O} \equiv \partial O / \partial \boldsymbol{\Phi}$ (i.e., $O_{\alpha} \equiv \partial O / \partial \Phi_{\alpha}$ ). One can then define the vector

$B_{\mu} \equiv \epsilon_{\mu \nu \lambda \rho \sigma \tau} O_{\nu} E_{\lambda}^{a} E_{\rho}^{b} E_{\sigma}^{c} E_{\tau}^{d}$

that characterises a direction in the space of CP-violating phases where there is no contribution to the observable $O$, nor to the EDMs. The EDM-free direction that optimises $O$ is clearly orthogonal to $B_{\mu}$ as well as to the EDM vectors $E_{\alpha}^{a, b, c, d}$. As such, it is characterised by the six-vector

$$
\begin{aligned}
\Phi_{\alpha} & =\epsilon_{\alpha \beta \gamma \delta \mu \eta} E_{\beta}^{a} E_{\gamma}^{b} E_{\delta}^{c} E_{\mu}^{d} B_{\eta} \\
& =\epsilon_{\alpha \beta \gamma \delta \mu \eta} \epsilon_{\eta \nu \lambda \rho \sigma \tau} E_{\beta}^{a} E_{\gamma}^{b} E_{\delta}^{c} E_{\mu}^{d} O_{\nu} E_{\lambda}^{a} E_{\rho}^{b} E_{\sigma}^{c} E_{\tau}^{d},
\end{aligned}
$$

with an unknown normalisation factor.

\subsection{Iterative geometric approach}

The linear geometric approach described above and used in [2] entails choosing a sample of points in the MSSM scenario of interest, fixing the phases to $0^{\circ}$ or $\pm 180^{\circ}$ for each scan point. Next one computes the optimal direction using the above geometric approach, and then one chooses randomly sets of phases along this direction. This is suitable as long as the phases are small, but we are also interested in the possibilities for large phases.

Here we use an iterative approach to extend and improve the efficiency of the linear geometric approach. After fixing the phases to $0^{\circ}$ or $\pm 180^{\circ}$ and computing the favoured direction with the geometric approach as discussed above, we move by $20^{\circ}$ along the favoured direction, and then recompute the favoured direction at this new position. This procedure is then iterated up to $100^{\circ}$.

\subsection{Sampling strategy}

We have generated several million points in each of the MSSM scenarios studied in the next section. Among those points, we have retained only those for which one of the neutral Higgs bosons has a mass in the range 121-129 GeV (corresponding to the measured value $\simeq 125 \mathrm{GeV}$ with a generous theoretical uncertainty), and we require the LSP to be the lightest neutralino. In addition, we impose the LEP and Tevatron SUSY mass limits and require squarks and the gluino to have masses above $500 \mathrm{GeV}$ as a conservative implementation of the LHC SUSY limits. Although the LHC SUSY search limits are stronger in more constrained MSSM scenarios, they become weaker in more general scenarios such as the pMSSM [16-18]. For consistency, here we apply the same loose constraints on the squark and gluino masses in all studied scenarios. Other constraints, such as those imposed by heavy-flavour, Higgs and direct dark matter measurements, are imposed at later stages in the analyses. 
The SUSY mass spectra and couplings, as well as the EDM constraints, are computed with CPsuperH [19-21]. The thorium monoxide EDM is calculated using the following formula [22]:

$$
\begin{aligned}
& d_{\mathrm{ThO}}[e . \mathrm{cm}] / \mathcal{F}_{\mathrm{ThO}}=d_{e}[e . \mathrm{cm}]+1.6 \\
& \times 10^{-21}[e . \mathrm{cm}] C_{S} \mathrm{TeV}^{2}+\cdots,
\end{aligned}
$$

where $d_{e}$ is the electron EDM and $C_{S}$ the coefficient of the $\mathrm{CP}$-odd electron nucleon interaction, which is also present in the thallium EDM. The left hand side of Eq. (5) is the quantity on which experimental constraints are provided currently [22]. Flavour constraints are calculated with SuperIso $[23,24]$ and CPsuperH. For the calculation of the dark matter relic density we used SuperIso Relic [25] and micrOMEGAs [26-28], and the later is also used for the calculation of scattering cross sections for dark matter direct detection. Finally, we use HiggsBounds [29] to assess the viability of the model points in view of the Higgs constraints.

\section{Studies of MSSM scenarios}

We now apply the approach described above to several representative MSSM scenarios.

\subsection{The CMSSM}

We first consider the CMSSM, in which the soft supersymmetry-breaking parameters $m_{0}, m_{1 / 2}$ and $A$ are each constrained to have universal values at an input grand-unification scale. This model is often analysed assuming some fixed value of $\tan \beta$, the ratio of Higgs v.e.v.s. Generalizing the usual CMSSM set-up, here we vary the 6 MSSM CP phases independently in order to allow more flexibility and a closer comparison with the other MSSM scenarios. Our startingpoint here is one of the best-fit CMSSM points found recently in a global analysis [11] of the $m_{0}, m_{1 / 2}, A, \tan \beta$ parameter space for the Higgsino mixing parameter $\mu>0$, neglecting all the possible MSSM sources of CP violation..$^{5}$ This point has

$$
\begin{aligned}
& m_{0}=670 \mathrm{GeV}, \quad m_{1 / 2}=1040 \mathrm{GeV}, \quad A=3440 \mathrm{GeV}, \\
& \tan \beta=21 .
\end{aligned}
$$

We use this point as a base for the geometric approach using the EDM limits in Table 1, treating the CP asymmetry in $b \rightarrow s \gamma, A_{\mathrm{CP}}$, as the observable to be maximised, in a followup of the study presented in [15]. We have generated more than 600000 sets of phases along the favoured direction, and

\footnotetext{
5 This analysis also found a high-mass best-fit point with a slightly lower value of the global $\chi^{2}$ function. However, the ATLAS jets + missing transverse energy constraint has subsequently been revised, and the low-mass point is now the global minimum of the $\chi^{2}$ function [12].
}

have found that about half of them pass the EDM constraints, which shows that the method is very efficient.

Figure 1 shows the distributions of the six CP-violating phases $\Phi_{\alpha}$ obtained from our sampling. The reader should bear in mind that these distributions have no 'probability' or 'likelihood' interpretation but only indicate how our iterative geometric procedure samples large values of the phases. We see that the effectiveness of the procedure differs significantly for different phases. We see that large values of $\Phi_{A_{b}}$ are relatively well sampled, whereas only intermediate $\Phi_{A_{t}}$ and $\Phi_{A_{\tau}}$ values can be reached, and we find no parameter sets with $\Phi_{1,2,3}$ substantially different from zero. This is because for the CMSSM best-fit point (6) it is not possible to cancel the contributions of the phases to all the EDMs simultaneously.

Figure 2 displays the results of this scan of the CPviolating CMSSM for the masses of the three neutral Higgs bosons $M_{h_{1}}, M_{h_{2}}, M_{h_{3}}$. The Higgs masses all lie in narrow ranges around their nominal values at the best-fit point in the CP-conserving CMSSM, namely $M_{h}=123 \mathrm{GeV}$, $M_{A} \simeq M_{H}=1410 \mathrm{GeV}$. In view of the theoretical uncertainties in calculating the Higgs masses for any specific set of CMSSM inputs, measuring Higgs masses would not constrain usefully the CP-violating parameters at the CMSSM best-fit point.

Figure 3 displays the results of this CMSSM scan for the $\mathrm{CP}$ asymmetry in $b \rightarrow s \gamma, A_{\mathrm{CP}}$, (left) and the spinindependent neutralino-proton scattering cross section $\sigma_{\mathrm{SI}}^{p} v$ (right). We find in this model values of $A_{\mathrm{CP}} \ll 10^{-3}$, which are considerably below the current and prospective experimental sensitivities. We conclude that the prospects for discovering the $A_{\mathrm{CP}}$ signature of $\mathrm{CP}$ violation in this particular CMSSM scenario are not good. Also, the spread in the values of $\sigma_{\mathrm{SI}}^{p} v$ is quite small, and much smaller than the theoretical uncertainties related to hadronic matrix elements and the astrophysical uncertainties in the local dark matter density, so this observable is also not a promising one for the CPviolating CMSSM.

We have also studied the possibility of a signature in $B_{s}$ meson mass mixing, with discouraging results. We have found that the new physics contribution, $\Delta M_{B_{s}}^{\mathrm{NP}}$ is always very small, namely $\sim 0.1 / \mathrm{ps}$, which is far below any prospective reduction in the uncertainty in the theoretical calculation of the contribution from the Standard Model [30]. Moreover, after applying the EDM constraints the CP-violating CMSSM contribution is forced to be exceedingly close to the value in the CP-conserving CMSSM.

In Fig. 4 we show scatter plots of $h_{1}$ signal strengths $\mu_{X}$ (normalised relative to the Standard Model values) in the best-fit CMSSM scenario (6) with non-zero CP-violating phases before (green dots) and after (blue dots) the EDM constraints. We see that the $\mathrm{CP}$-violating case expands the ranges of these observables found already in the $\mathrm{CP}$-conserving case, in particular after imposing the EDM constraints. However, 

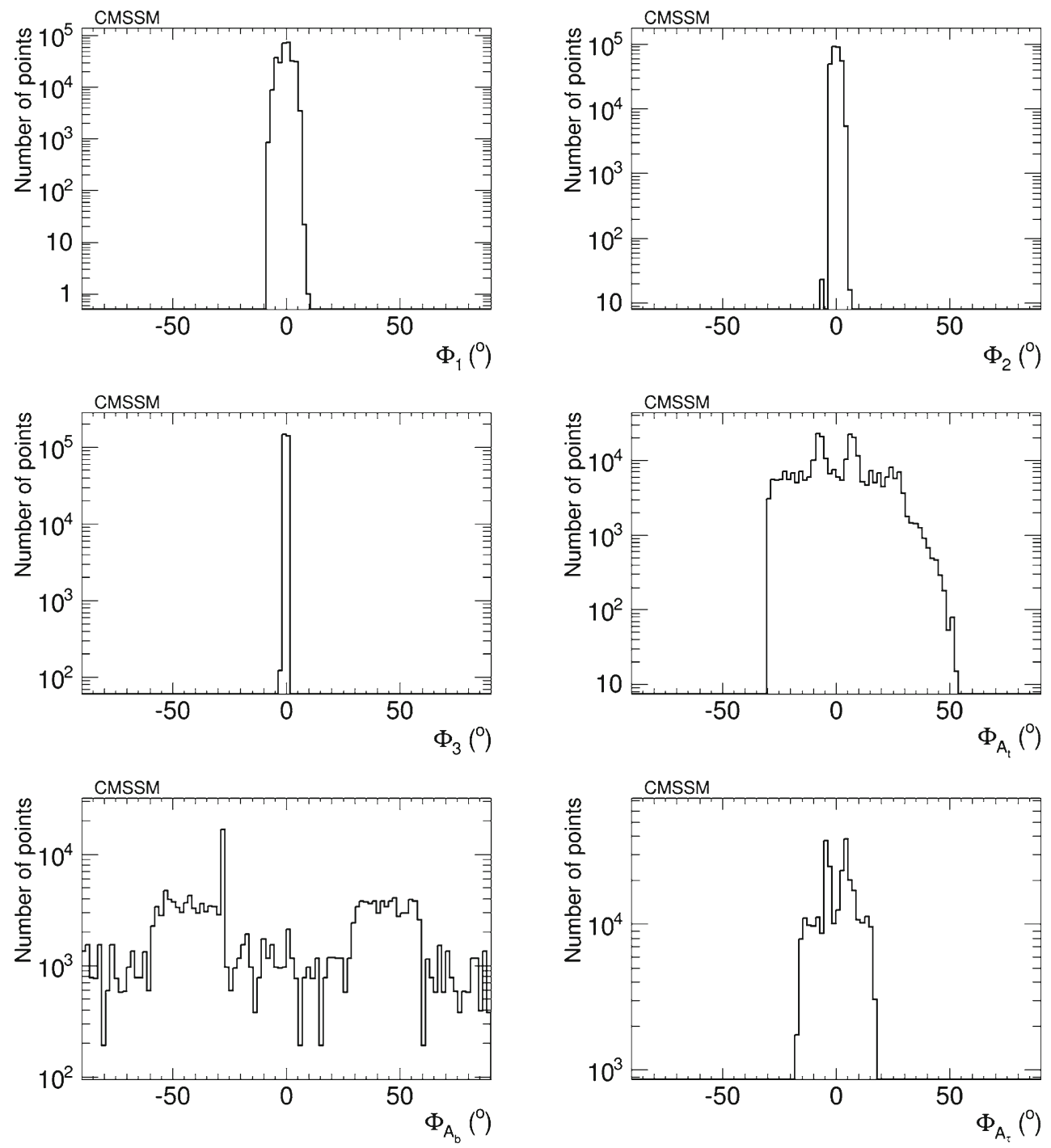

Fig. 1 Sampling of the CP-violating phases $\Phi_{\alpha}$ in the best-fit CMSSM scenario (6) generated in the iterative geometric approach, imposing the EDM limits as well as constraints from the cosmological cold dark

these expanded ranges all lie well within the current experimental uncertainties. In the left panel we see a strong, almost linear correlation between $\mu_{\gamma \gamma}$ and $\mu_{g g}$, which becomes milder in the right panel, between $\mu_{V V}$ and $\mu_{\bar{b} b}$. The signal strengths are close to but smaller than unity.

We emphasise that the Higgs couplings to fermions provide the most unambiguous probe of its $\mathrm{CP}$ properties, even more so when there is $\mathrm{CP}$ mixing, as the Higgs may couple to the $\mathrm{CP}$-even and $\mathrm{CP}$-odd fermion states in a democratic manner. In cases of the $h_{i} V V$ couplings, there are two effects of CP mixing in the Higgs sector. One is a reduction in the strength of coefficient of the $g_{\mu \nu}$ term in the $h_{i} V V$ vertex and thus in the rates. The second is the simultaneous presence of the $\mathrm{CP}$-even and $\mathrm{CP}$-odd tensor structures in the vertex. The coefficient of the CP-odd term in the $h_{i} V V$ vertex involving matter relic density and upper limits on direct detection, from flavour physics and from Higgs searches

the $\epsilon_{\mu v \rho \lambda}$ tensor is by necessity small, as it is always loopinduced. Reduction in the production rates is reflected in signal strengths, but these, while currently providing the best available information, are necessarily ambiguous, as there are other mechanisms that may lead to the rate modification. On the other hand, since the fermions couple democratically to the CP-even and CP-odd parts of the Higgs couplings, ascertaining the simultaneous presence of $\bar{f} f h_{i}$ and $\bar{f} \gamma_{5} f h_{i}$ terms in the vertex through various angular distributions and kinematic variables is unambiguous.

We have therefore analysed the prospects for $\mathrm{CP}$ violation in the couplings of the neutral Higgs bosons to $\tau^{+} \tau^{-}$and $\bar{t} t$, by calculating the quantities $\phi_{\tau}^{h_{i}}$ and $\phi_{t}^{h_{i}}$ for $i=1,2,3$, which are expressed in terms of the corresponding pseudoscalar and scalar couplings by 

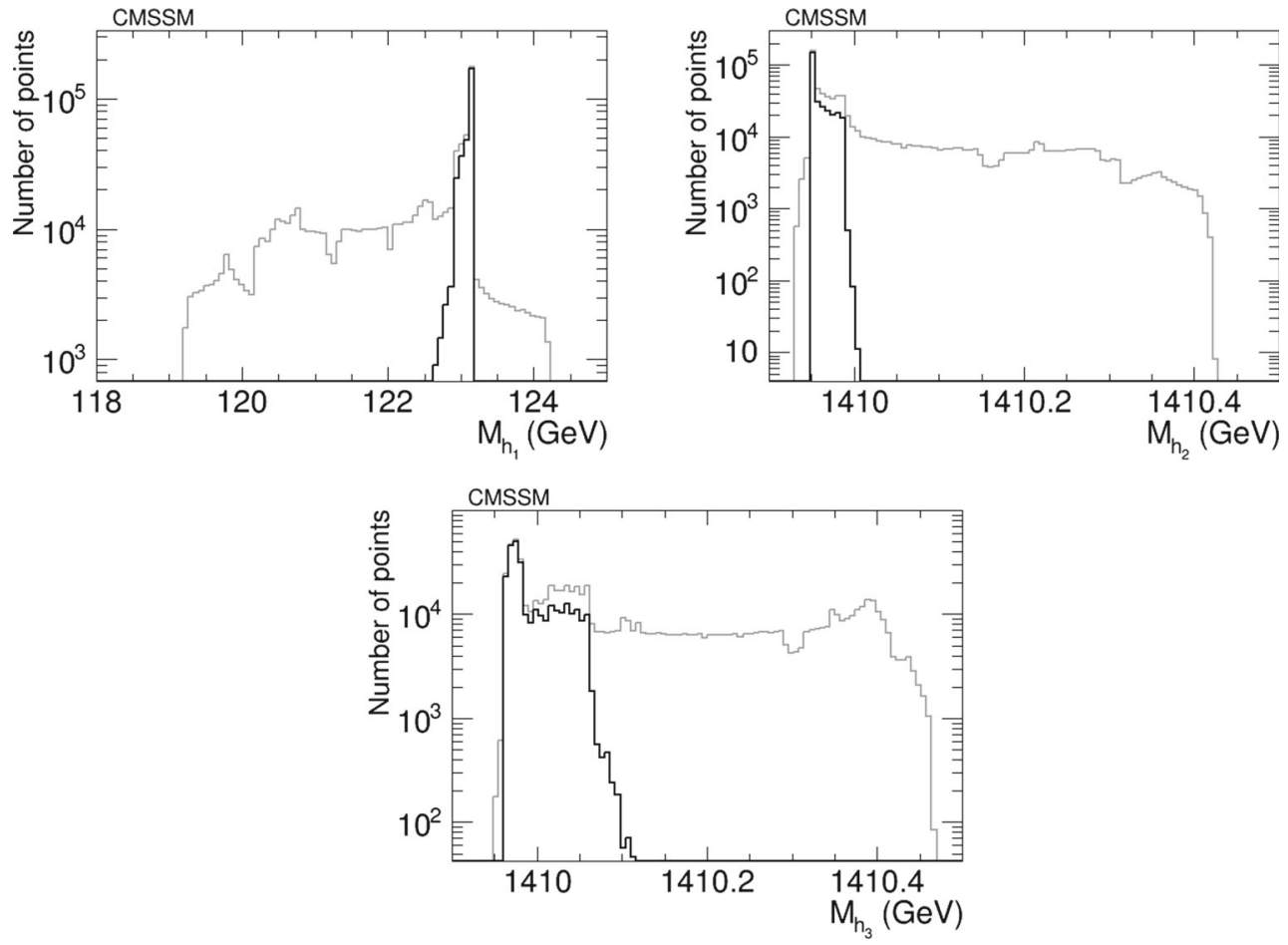

Fig. 2 The distributions of the three Higgs masses $M_{h_{1}}, M_{h_{2}}, M_{h_{3}}$ in the CP-violating CMSSM before (grey) and after (black) applying the EDM constraints using the geometric approach described in the text, assuming the best-fit values of $m_{0}, m_{1 / 2}, A$ and $\tan \beta$ (6) found in a global analysis

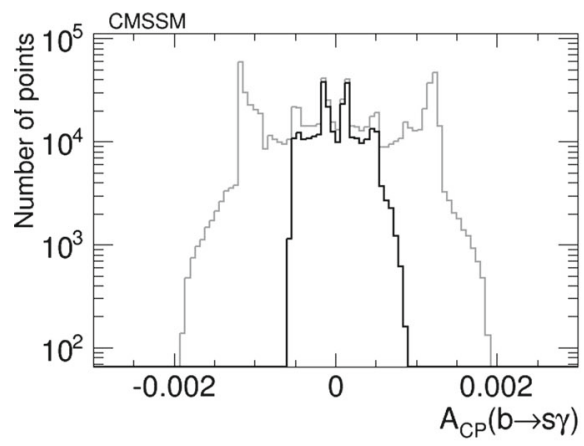

Fig. 3 The distributions of (left) the CP asymmetry in $b \rightarrow s \gamma, A_{\mathrm{CP}}$, in the CMSSM and (right) the spin-independent scattering cross section for neutralino scattering on protons, found before (grey) and after

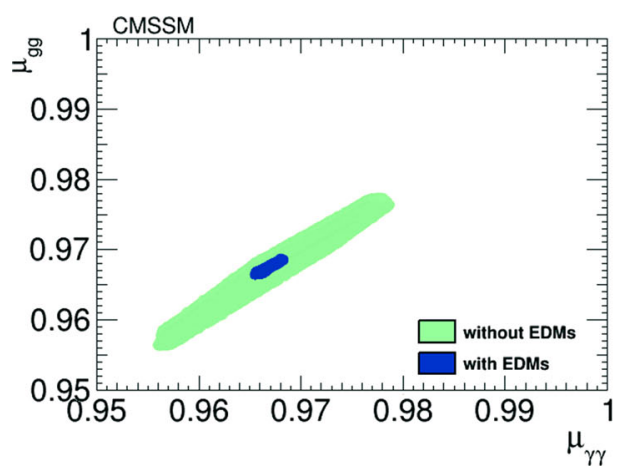

Fig. 4 Scatter plots of the $h_{1}$ signal strengths in the best-fit CMSSM scenario (6) in the CP-violating limit before (green dots) and after (blue dots) imposing the EDM constraints. The left panel displays a strong

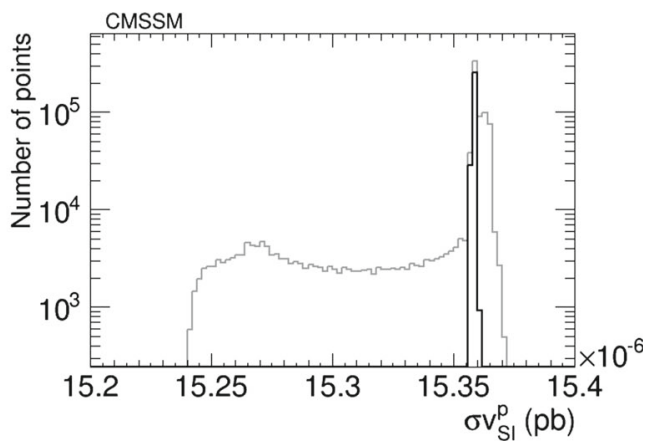

(black) applying the EDM constraints using the geometric approach described in the text and assuming the best-fit values of $m_{0}, m_{1 / 2}, A$ and $\tan \beta$ (6) found in a global analysis $[11,12]$

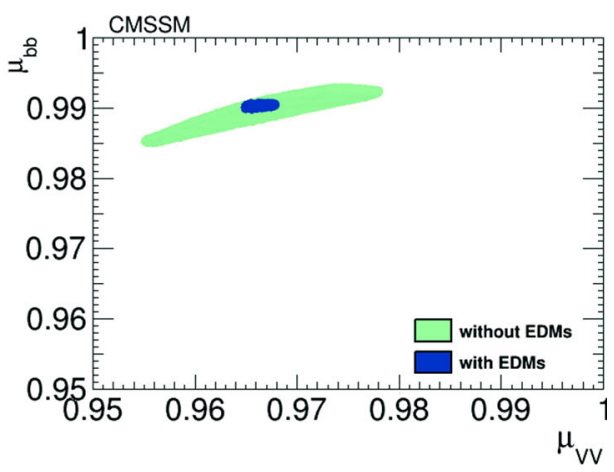

linear correlation between $\mu_{\gamma \gamma}$ and $\mu_{g g}$, and the right panel displays the correlation between $\mu_{V V}$ and $\mu_{\bar{b} b}$ 


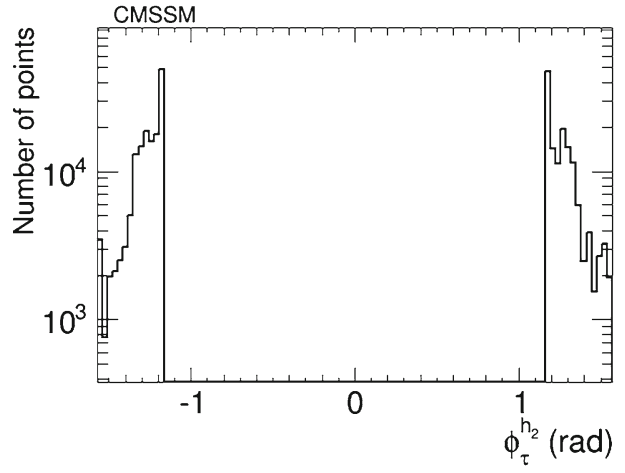

Fig. 5 The distributions of (left) the CP-violating phase $\phi_{\tau}^{h_{2}}$ in $h_{2} \tau \tau$ couplings and (right) the CP-violating phase $\phi_{\tau}^{h_{3}}$ in $h_{3} \tau \tau$ couplings in the CMSSM, found after applying the EDM constraints using the geo-

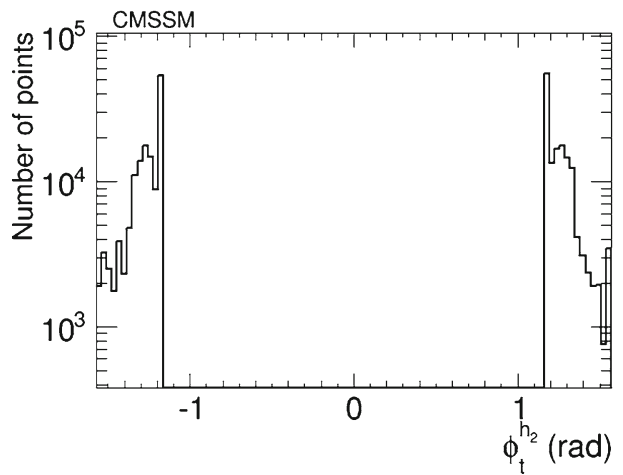

Fig. 6 The distributions of (left) the CP-violating phase $\phi_{t}^{h_{2}}$ in $h_{2} \bar{t} t$ couplings and (right) the CP-violating phase $\phi_{t}^{h_{3}}$ in $h_{3} \bar{t} t$ couplings in the CMSSM, found after applying the EDM constraints using the geo-

$\tan \phi_{\tau}^{h_{i}} \equiv \frac{g_{P}^{h_{i} \tau \tau}}{g_{S}^{h_{i} \tau \tau}}, \quad \tan \phi_{t}^{h_{i}} \equiv \frac{g_{P}^{h_{i} \bar{t} t}}{g_{S}^{h_{i} \bar{t} t}}$

After imposing the EDM constraints, we find that the phases for the $h_{1}$ couplings are very small, $\lesssim 0.02$ radians. On the other hand, the phases for the $h_{2}$ and $h_{3}$ couplings may be quite large, as seen in Figs. 5 and 6, respectively. The $h_{2}$ couplings have phases close to $\pm \pi$, corresponding to a mainly CP-odd state, while the $h_{3}$ couplings are close to 0 corresponding to a mainly $\mathrm{CP}$-even state. A detailed discussion of the prospects for measuring these phases at the LHC and/or future colliders lies beyond the scope of this work. Clearly, any such future analysis would need to take into account the near-degeneracy of the $h_{2}$ and $h_{3}$ bosons, as seen in Fig. 2, whose implications would be different for $p p, e^{+} e^{-}, \mu^{+} \mu^{-}$ and $\gamma \gamma$ colliders.

We limit ourselves to pointing out a few of these. In the case of the light Higgs, associated production of Higgs with a $\bar{t} t$ pair or a single $t$ or $\bar{t}$ can be used for this [31,32]. However, in our case since it is the heavier Higgses that have the larger $\mathrm{CP}$ violation, associated production may not be the best way, but decays of the Higgs into a $\tau$ pair (or even into a $\bar{t} t$ pair

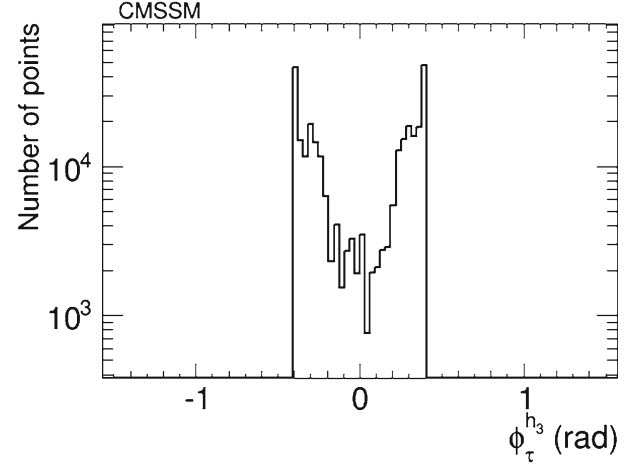

metric approach described in the text and assuming the best-fit values of $m_{0}, m_{1 / 2}, A$ and $\tan \beta$ (6) found in a global analysis [11,12]

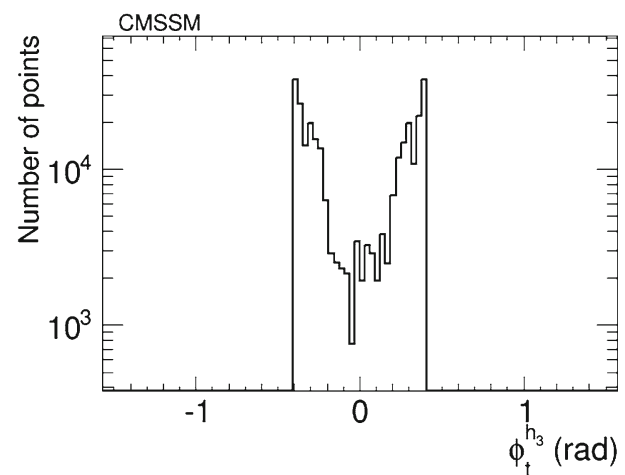

metric approach described in the text and assuming the best-fit values of $m_{0}, m_{1 / 2}, A$ and $\tan \beta$ (6) found in a global analysis [11,12]

if the Higgs is heavy enough) and analysis of the spins of the decay $\tau / t$ can be used at $\gamma \gamma$ colliders [33-36] and even at the LHC [37-39]. The method of Ref. [37] is particularly promising when the $h_{2,3}$ are degenerate.

\subsection{NUHM2}

We now use the iterative geometric approach with four EDM constraints to analyse CP violation in the NUHM2 scenario, in which the gaugino masses, trilinear couplings and soft supersymmetry-breaking contributions to the squark and slepton masses $M_{\tilde{f}}$ are universal, but those to the two Higgs doublets are allowed to vary independently. The freedom in these two parameters can be traded via the electroweak vacuum conditions for free values of $\mu$ and a heavy Higgs mass parameter: to avoid complications with the three-way CPviolating mixing in the neutral sector, we take this second free parameter to be $m_{H^{ \pm}}$. We perform a random scan over the following ranges of the NUHM2 mass parameters:

$$
M_{1}=M_{2}=M_{3}=m_{1 / 2} \in[50,3000] \mathrm{GeV},
$$

$A_{0} \in[0,10000] \mathrm{GeV}$, 

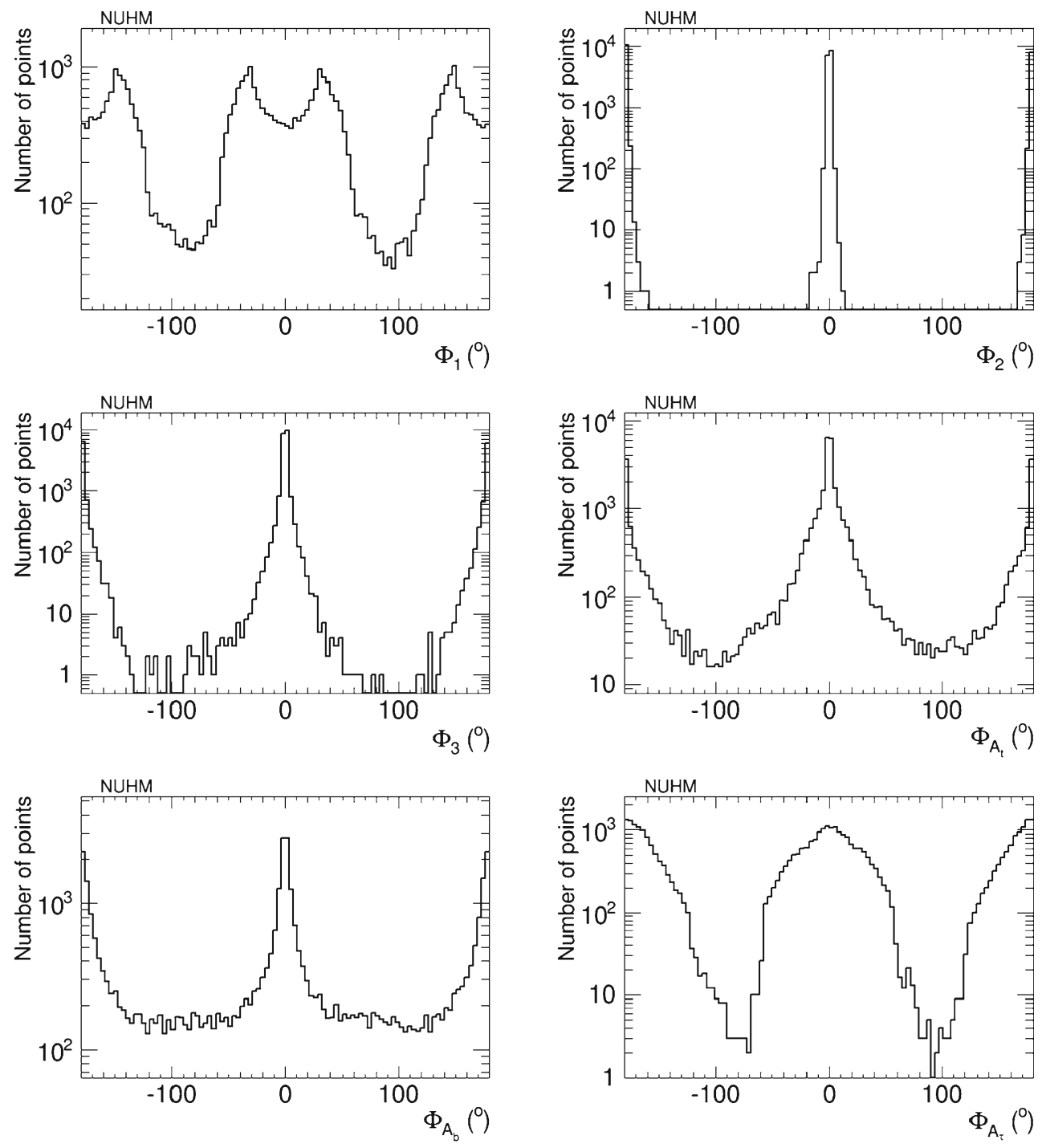

Fig. 7 Sampling of the CP-violating phases $\Phi_{\alpha}$ in the NUHM2 scenario generated in the iterative geometric approach, imposing the EDM and other constraints

$$
\begin{aligned}
M_{\tilde{f}} & =m_{0} \in[50,3000] \mathrm{GeV}, \quad m_{H^{ \pm}} \in[1,2000] \mathrm{GeV}, \\
\mu & \in[-2000,2000] \mathrm{GeV},
\end{aligned}
$$

with $\tan \beta \in[1,60]$ and varying the six phases $\Phi_{\alpha}$ independently as before, using the geometrical approach to seek maximal values of $A_{\mathrm{CP}}$.

Figure 7 displays the samples of the six CP-violating phases $\Phi_{\alpha}$ obtained in our analysis. We see that our iterative geometrical approach enables us to sample effectively large values of $\Phi_{1}, \Phi_{A_{t}}$ and $\Phi_{A_{b}}$, whereas large values of $\Phi_{3}$ and $\Phi_{A_{\tau}}$ are sampled less effectively, and we do not find large values of $\Phi_{2}$. Again, we emphasise that these distributions do not have any 'probability' or 'likelihood' interpretation. However, the absence of large values of $\Phi_{2}$ indicates that there is no way to cancel the contributions of this and the other phases to all the EDMs simultaneously.
Figure 8 provides a visualisation of the cancellations that are required to respect the EDM constraints. In the left panel we see the correlation these constraints impose between $\Phi_{3}$ and $\Phi_{A_{t}}$, and in the right panel the correlation between $\Phi_{3}$ and $\Phi_{A_{b}}$. In both cases we see diagonal features corresponding to close correlations, but we also see populations of points with large phases, e.g., in the neighbourhood of $\left(\Phi_{A_{t}}, \Phi_{3}\right) \sim\left(90^{\circ},-90^{\circ}\right)$ in the left panel, and extending to $\left(\Phi_{A_{b}}, \Phi_{3}\right) \sim\left(-90^{\circ},-90^{\circ}\right)$ in the right panel. These examples serve as reminders that the EDM constraints do not require all the $\mathrm{CP}$-violating phases to be small simultaneously.

The iterative geometric approach was designed to find the maximal values of the CP-violating asymmetry in $b \rightarrow s \gamma$ decays, $A_{\mathrm{CP}}$, that are compatible with the EDM constraints. We see in the left panel of Fig. 9 that values of $A_{\mathrm{CP}} \lesssim 2 \%$ can 

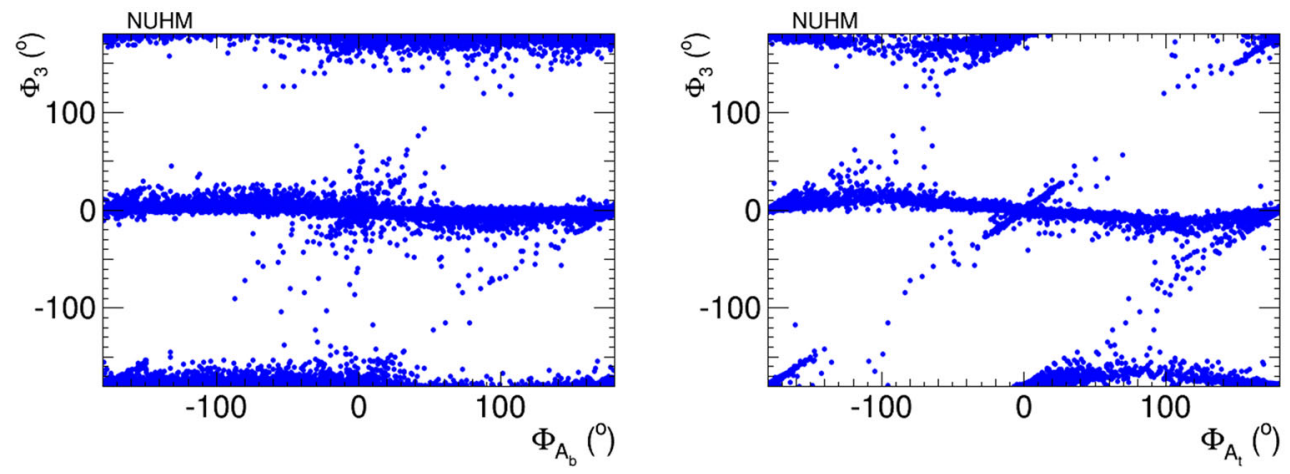

Fig. 8 Correlations of $\Phi_{3}$ with $\Phi_{\mathrm{A}_{\mathrm{b}}}$ (left panel) and $\Phi_{3}$ with $\Phi_{\mathrm{A}_{\mathrm{t}}}$ (right panel) imposed by the EDM constraints in the NUHM2 scenario

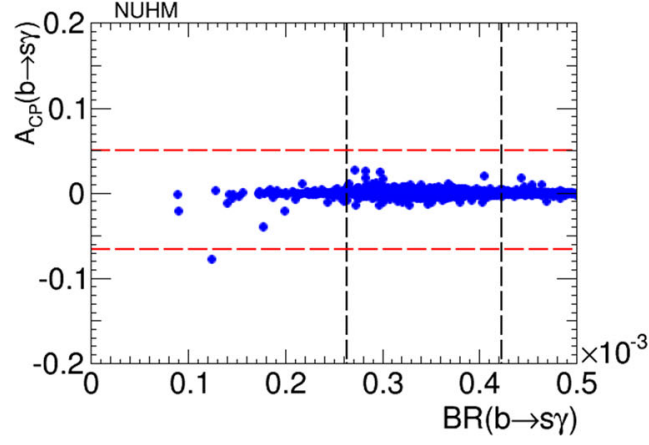

Fig. 9 Left panel Scatter plot of the branching ratio for $b \rightarrow s \gamma$ decay versus its $\mathrm{CP}$-violating asymmetry, $A_{\mathrm{CP}}$, in the NUHM2 scenario. Right panel Histogram of $A_{\mathrm{CP}}$ in the NUHM2, imposing only the Higgs mass and EDM cuts ( grey full sample, black points satisfying the EDM con-

be found in the NUHM2 for values of the $b \rightarrow s \gamma$ branching ratio lying within the experimentally allowed range. The right panel of Fig. 9 displays a histogram of these results for the NUHM2 (grey: full sample, black: points satisfying the EDM constraints). The present experimental constraints on $A_{\mathrm{CP}}$ are shown as vertical red dashed lines [40], and the vertical green dashed lines represent the possible future improvement in the experimental sensitivity by a factor of 10 , corresponding to the prospective Belle II sensitivity [41]. We see that there are CP-violating NUHM2 models that could be explored with such an improvement: the EDM constraints do not exclude an observable value of $A_{\mathrm{CP}}$, and such a measurement would provide additional information on $\mathrm{CP}$ violation within the NUHM2.

We have also calculated the possible new physics contribution to $B_{s}$ meson mass mixing, $\Delta M_{B_{s}}^{\mathrm{NP}}$, in the NUHM2 scenario, as shown in Fig. 10. The grey histogram is for the full sample of NUHM2 points satisfying the Higgs mass and other constraints, and the black histogram is for points that also satisfy the EDM constraints. The present experimental upper limit on $\Delta M_{B_{s}}^{\mathrm{NP}}$ is shown as the vertical red dashed line [40]. The vertical yellow dashed line in Fig. 10 represents the possible sensitivity if the theoretical uncertainty in the Standard Model contribution to $B_{s}$ mixing could be reduced by

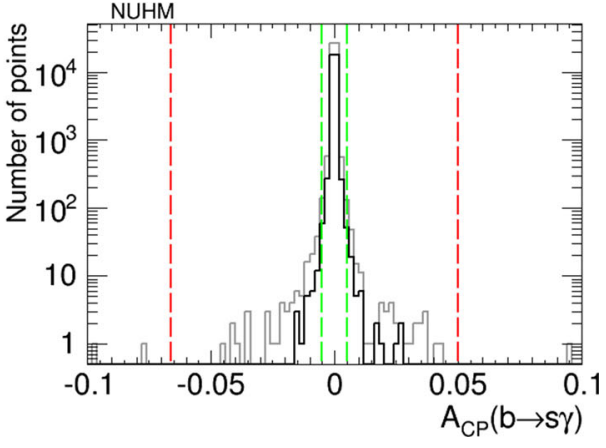

straints). The vertical red dashed lines represent the present experimental limits, and the vertical green dashed lines represent the prospective future improvement in the sensitivity to $A_{\mathrm{CP}}$ by a factor of 10

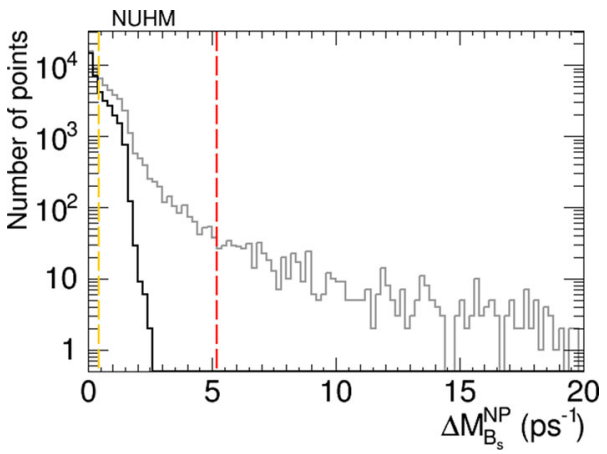

Fig. 10 Histogram of the possible new physics contribution to $B_{S}$ mixing, $\Delta M_{B_{s}}^{\mathrm{NP}}$, in the NUHM2 scenario. The grey histogram is for points satisfying the Higgs mass and other constraints, and the black histogram is for points that also satisfy the EDM constraints. The vertical red dashed line is the present experimental upper limit on $\Delta M_{B_{s}}^{\mathrm{NP}}$, and the vertical yellow dashed line shows the potential of a reduction in the current theoretical uncertainty in the Standard Model by a factor of 10

a factor of 10 thanks to improved lattice calculations. In this case, many of the viable NUHM2 models (indicated by the black histogram) could be explored.

We have not imposed a priori consistency with the cosmological constraints on the relic LSP density $\Omega_{\chi} h^{2}$ and the 


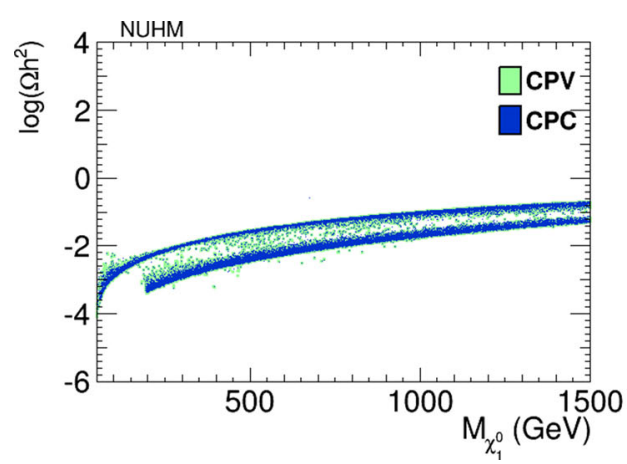

Fig. 11 Left panel Scatter plot of the dark matter relic density as a function of the neutralino mass in the NUHM2 scenario. Right panel Scatter plot of the spin-independent dark matter scattering cross section

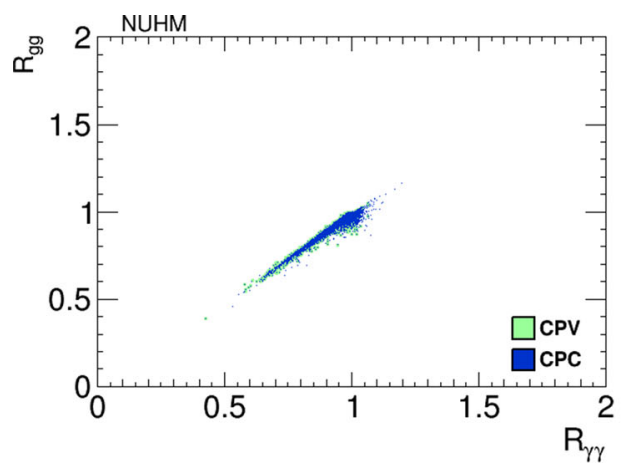

Fig. 12 Scatter plots of branching ratios, normalised to the Standard Model values, for decays of the lightest Higgs boson, $h_{1}$, in the NUHM2 scenario in the CP-violating limit $\Phi_{\alpha}=0$ (blue dots) and in the CP-

spin-independent dark matter scattering cross section $\sigma_{\mathrm{SI}}^{p} v$. As we see in the left panel of Fig. 11, the values of the relic density for the CP-violating NUHM2 (green points) are very similar to those in the CP-conserving version (blue points), and they are generally within the range allowed for a supersymmetric contribution to the dark matter density. The right panel of Fig. 11 shows that the values of $\sigma_{\mathrm{SI}}^{p} v$ are also rather similar, with some differences for low cross-section values well below the experimental upper limit from LUX [42], which is shown as the black solid line.

Figure 12 shows scatter plots of values of $h_{1}$ branching ratios in the NUHM2 scenario. The left panel displays $\left(R_{\gamma \gamma}, R_{g g}\right)$ and the right panel displays $\left(R_{V V}, R_{\bar{b} b}\right)$. The blue dots are CP-conserving parameter choices with $\Phi_{\alpha}=0$, and the green dots are from a scan of CP-violating points with $\Phi_{\alpha} \neq 0$. We note in the left panel a strong correlation between $R_{\gamma \gamma}$ and $R_{g g}$, which may be either much smaller than in the Standard Model or somewhat larger, which is due to the variation of the Higgs width induced by a modification of the Higgs to $\bar{b} b$ branching fraction. ${ }^{6}$ We see in the right

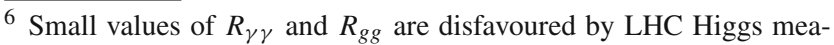
surements.
}

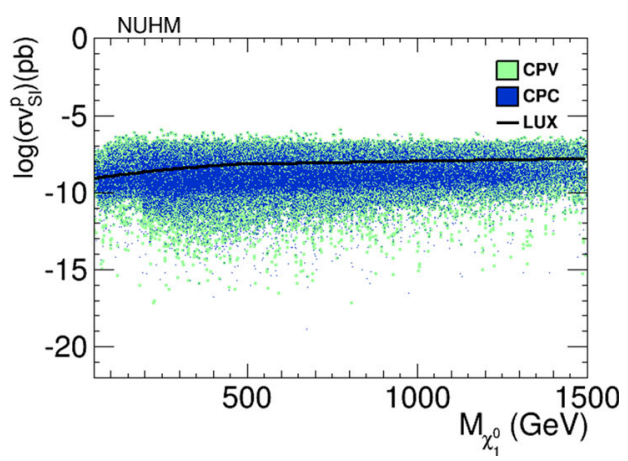

$\sigma_{\mathrm{SI}}^{p} v$ as a function of the neutralino mass in the NUHM2 scenario. In both panels, CP-conserving parameter choices are denoted by blue dots, and CP-violating parameter choices by green dots

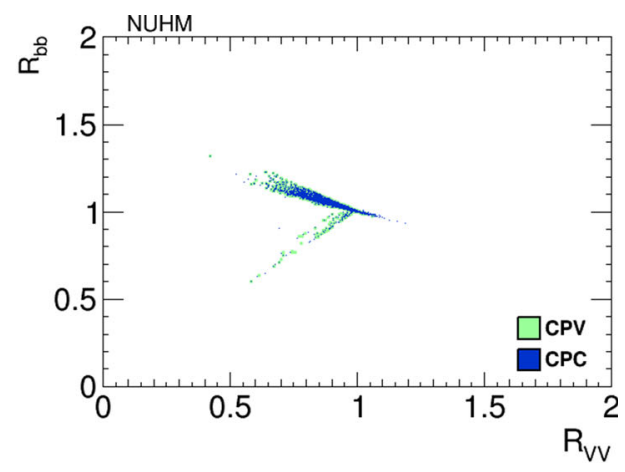

violating sample (green dots). The left panel displays a linear correlation between $R_{\gamma \gamma}$ and $R_{g g}$, and the right panel displays a bimodal correlation between $R_{V V}$ and $R_{\bar{b} b}$

panel that a large reduction in $R_{V V}$ is also possible, which may be accompanied by values of $R_{\bar{b} b}$ that are either larger or smaller than in the Standard Model. The branch with larger values of $R_{\bar{b} b}$ is also related to the variation of the Higgs width, while the points corresponding to a decrease of both ratios are due to an enhancement of decays to light SUSY particles [43-45]. ${ }^{7}$

Scatter plots of $h_{1}$ signal strengths $\mu_{X}$ in the NUHM2 scenario with the CP-violating phases $\Phi_{\alpha}=0$ (blue dots) and $\neq 0$ (green dots) are shown in Fig. 13.

We see a strong, almost linear correlation between $\mu_{\gamma \gamma}$ and $\mu_{g g}$ in the left panel, and in the right panel we see a correlation between $\mu_{V V}$ and $\mu_{\bar{b} b}$ that is bimodal for small values of $\mu_{V V}$. No significant difference is observed between the $\mathrm{CP}$-conserving and the $\mathrm{CP}$-violating cases.

The prospects for $\mathrm{CP}$ violation in the couplings of the heavy neutral Higgs bosons to $\tau^{+} \tau^{-}$and $\bar{t} t$ in the NUHM2 scenario (8) are shown in Figs. 14 and 15. As in the CMSSM case discussed previously, we find that after imposing all the constraints the phases for the $h_{1}$ couplings are small, namely

\footnotetext{
7 Small values of $R_{V V}$ are disfavoured by LHC Higgs measurements, whereas a relatively large range of $R_{\bar{b} b}$ is still allowed.
} 


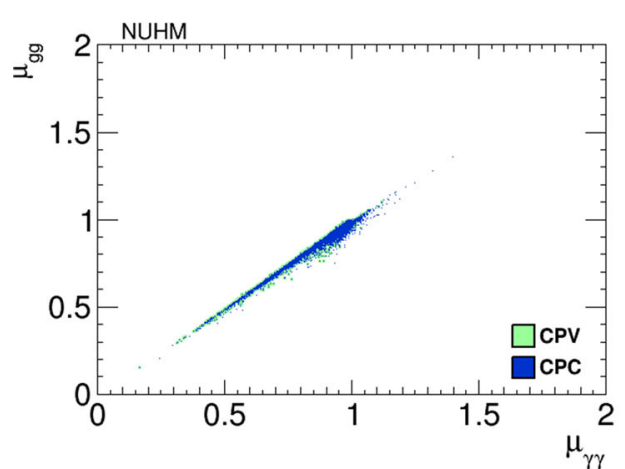

Fig. 13 Scatter plots of the $h_{1}$ signal strengths in the NUHM2 scenario in the CP-violating limit $\Phi_{\alpha}=0$ (blue dots) and in the CP-violating sample (green dots). The left panel displays a strong linear correlation

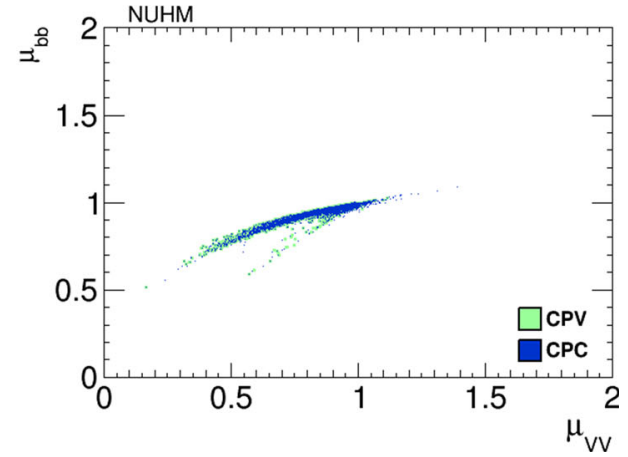

between $\mu_{\gamma \gamma}$ and $\mu_{g g}$, and the right panel displays a bimodal correlation between $\mu_{V V}$ and $\mu_{\bar{b} b}$

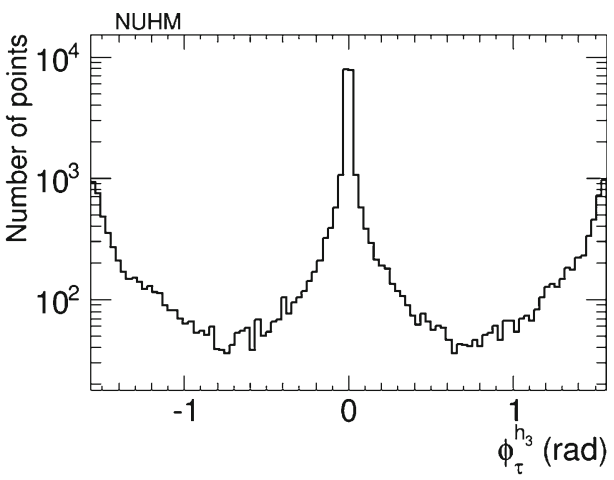

Fig. 14 The distributions of (left) the CP-violating phase $\phi_{\tau}^{h_{2}}$ in $h_{2} \tau \tau$ couplings and (right) the CP-violating phase $\phi_{\tau}^{h_{3}}$ in $h_{3} \tau \tau$ couplings in the NUHM2 scenario (8), found after applying all the constraints using the geometric approach described in the text
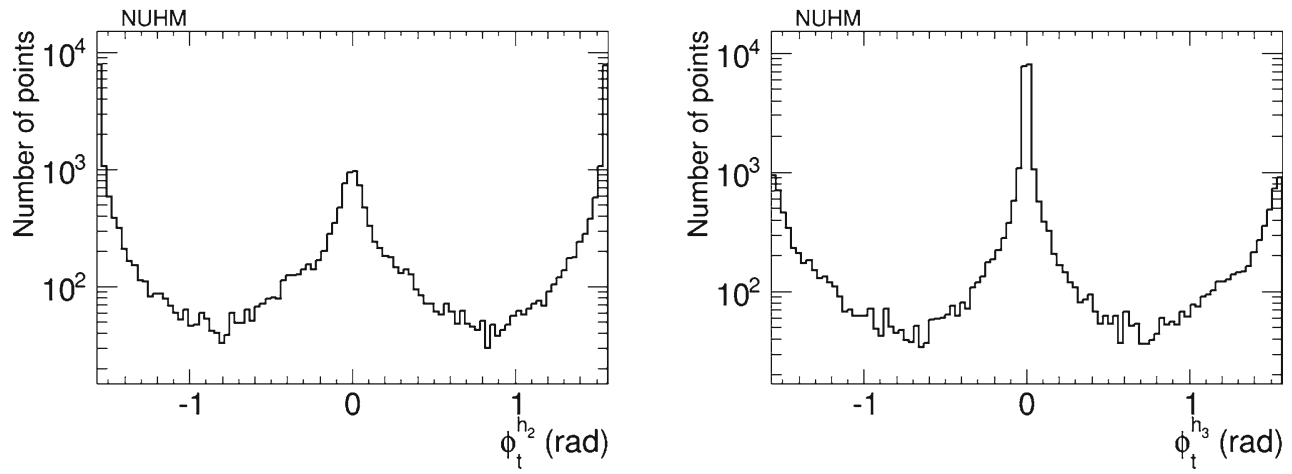

Fig. 15 The distributions of (left) the CP-violating phase $\phi_{t}^{h_{2}}$ in $h_{2} \bar{t} t$ couplings and (right) the CP-violating phase $\phi_{t}^{h_{3}}$ in $h_{3} \bar{t} t$ couplings in the NUHM2 scenario (8), found after applying all the constraints using the geometric approach described in the text

$\lesssim 0.02$ radians. On the other hand, $h_{2,3}$ decays may provide interesting prospects for probing $\mathrm{CP}$ violation also in this NUHM2 scenario.

\subsection{CPX}

We now apply the iterative geometric approach with four EDMs and one CP-violating observable described earlier to a CPX scenario in which
$M_{Q_{3}}=M_{U_{3}}=M_{D_{3}}=M_{L_{3}}=M_{E_{3}} \equiv M_{S}$,

$\mu=4 M_{S}, \quad\left|A_{t, b, \tau}\right|=2 M_{S}, \quad\left|M_{1,2}\right|=1 \mathrm{TeV}$,

$$
\left|M_{3}\right|=3 \mathrm{TeV} \text {, }
$$

performing random scans over the following parameter ranges:

$M_{S} \in[50,3000] \mathrm{GeV}, \quad m_{H^{ \pm}} \in[1,2000] \mathrm{GeV}$,

$\tan \beta \in[1,60]$, 

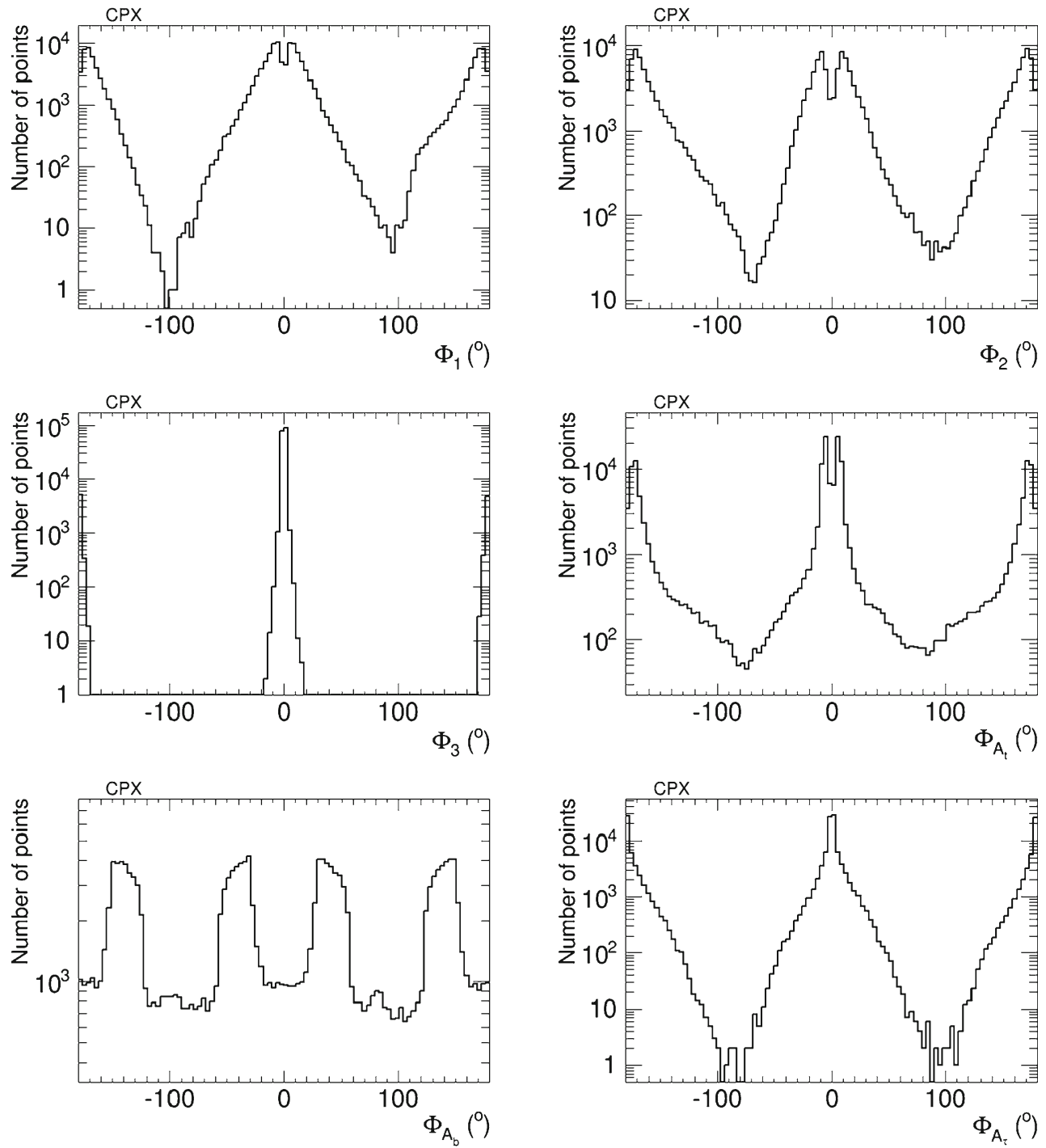

Fig. 16 Sampling of the CP-violating phases $\Phi_{\alpha}$ in the CPX scenario generated in the iterative geometric approach, imposing the EDM and other constraints

with the six CP-violating phases of the MCPMFV model being considered independent, as before.

Figure 16 displays the distributions of the six CP-violating phases $\Phi_{\alpha}$ sampled in our analysis. We emphasise that these distributions do not have any 'probability' or 'likelihood' interpretation. Rather, they serve to indicate how well our iterative geometric procedure gives access to large values of the phases that are difficult to sample in a simple random scan, because of the cancellations required to bring the EDMs within the allowed ranges shown in Table 1 . We see that the effectiveness of the procedure differs significantly for different phases. For example, in the case of $\Phi_{A_{b}}$ our procedure yields almost as many parameter sets with $\Phi_{A_{b}} \sim \pm 90^{\circ}$ as with $\Phi_{A_{b}} \sim 0^{\circ}$ or $180^{\circ}$, and actually yields more parameter sets with intermediate values of $\Phi_{A_{b}}$. In the case of $\Phi_{A_{t}}$, the procedure yields a factor $\sim 100$ lower sampling den- sity for $\Phi_{A_{b}} \sim \pm 90^{\circ}$ than for $\Phi_{A_{b}} \sim 0^{\circ}, 180^{\circ}$, and larger factors for $\Phi_{2}, \Phi_{1}$ and $\Phi_{A_{\tau}}$. Finally, we find no parameter sets for $\Phi_{3} \sim \pm 90^{\circ}$ : this is because (for the choices of soft supersymmetry-breaking parameters in (9)) there is no way to cancel the contributions of this and the other phases to all the EDMs simultaneously.

In the CPX scenario we do not find values of $A_{\mathrm{CP}}$ that are large enough to be observable in the foreseeable future. However, we do find a possible signature in the new physics contribution to $B_{s}$ meson mass mixing, $\Delta M_{B_{s}}^{\mathrm{NP}}$, as shown in Fig. 17. The grey histogram is for CPX points satisfying the Higgs mass and other constraints, and the black histogram is for points that also satisfy the EDM constraints, including the present experimental upper limit on $\Delta M_{B_{s}}^{\mathrm{NP}}$, which is shown as the vertical red dashed line. The magnitude of this upper limit is largely due to the theoretical uncertainty in the 


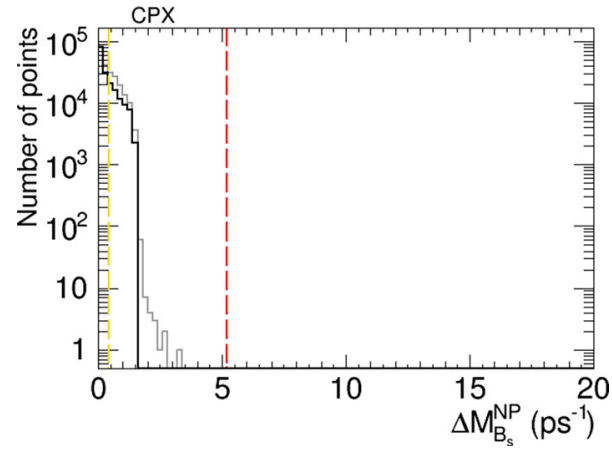

Fig. 17 Histogram of the possible new physics contribution to $B_{S}$ mixing, $\Delta M_{B_{s}}^{\mathrm{NP}}$, in the CPX scenario. The grey histogram is for points satisfying the Higgs mass and other constraints, and the black histogram is for points that also satisfy the EDM constraints. The vertical red dashed line is the present experimental upper limit on $\Delta M_{B_{S}}^{\mathrm{NP}}$, and the vertical yellow dashed line shows the potential of a reduction in the current theoretical uncertainty in the Standard Model by a factor of 10

Standard Model contribution to $B_{s}$ mixing, which is in turn associated with lattice calculations. If this uncertainty could be reduced by a factor of 10 , the sensitivity to new physics in $B_{S}$ mixing would become that indicated by the vertical yellow dashed line in Fig. 17, which could explore many of the CPX models indicated by the black histogram.

We display in Fig. 18 scatter plots of values of branching ratios in the CPX scenario of the lightest Higgs boson, $h_{1}$, normalised relative to the Standard Model values. The left panel shows $\left(R_{\gamma \gamma}, R_{g g}\right)$ and the right panel shows $\left(R_{V V}, R_{\bar{b} b}\right)$ in the limits where the phases $\Phi_{\alpha}=0$ (blue dots) and scanning over the values of $\Phi_{\alpha} \neq 0$ allowed by the EDMs (green dots). There are very small differences between the values of these quantities found in the $\mathrm{CP}$-conserving and CP-violating samples. In both cases, correlated substantial reductions in $R_{\gamma \gamma}$ and $R_{g g}$ are possible, as is a large reduction in $R_{V V}$ relative to the Standard Model value. On the other hand, the 'Cuba'-shaped plot in the right panel shows that $R_{\bar{b} b}$ is anti-correlated with $R_{V V}$, and may be enhanced to $\sim 1.3$ times the Standard Model value.

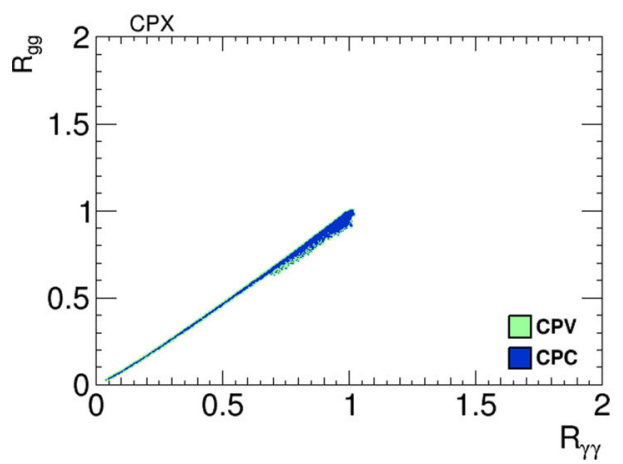

Fig. 18 Scatter plots of branching ratios, normalised to the Standard Model values, for decays of the lightest Higgs boson, $h_{1}$, in the CPX scenario in the CP-violating limit $\Phi_{\alpha}=0$ (blue dots) and in the CP-
Figure 19 shows scatter plots of $h_{1}$ signal strengths $\mu_{X}$ in the CPX scenario with the CP-violating phases $\Phi_{\alpha}=$ 0 (blue dots) and $\neq 0$ (green dots): again only very small differences are seen. In the left panel we see a strong, almost linear correlation between $\mu_{\gamma \gamma}$ and $\mu_{g g}$, and in the right panel we see a nonlinear correlation between $\mu_{V V}$ and $\mu_{\bar{b} b}$.

As already mentioned, our results for $A_{\mathrm{CP}}$ in the CPX scenario are very small, so we do not display them. Taken together with the results shown in Figs. 18 and 19, where no distinctive signatures of non-zero phases $\Phi_{\alpha} \neq 0$ are visible, our results suggest that one should look elsewhere for probes of $\mathrm{CP}$ violation in the $\mathrm{CPX}$ scenario.

We have also analysed the prospects for $\mathrm{CP}$ violation in the couplings of the neutral Higgs bosons to $\tau^{+} \tau^{-}$and $\bar{t} t$ in the CPX scenario $(9,10)$, as given by the phases $\phi_{\tau}^{h_{i}}$ and $\phi_{t}^{h_{i}}$ for $i=1,2,3$ defined in (7). As in the CMSSM case discussed previously, we find that after imposing the EDM constraints the phases for the $h_{1}$ couplings are small, $\phi_{\tau}^{h_{i}} \lesssim 0.1$ radians and $\phi_{t}^{h_{i}} \lesssim 0.02$ radians. On the other hand, the phases for the $h_{2}$ and $h_{3}$ couplings may again be quite large, as seen in Figs. 20 and 21, respectively. Thus $h_{2,3}$ decays may also provide interesting prospects for probing $\mathrm{CP}$ violation in this CPX scenario.

\subsection{Phenomenological MSSM (pMSSM)}

We now consider the MCPMFV version of the phenomenological MSSM (pMSSM), which has 25 parameters: the 19 real parameters

$$
\begin{aligned}
& M_{1,2,3}, M_{\tilde{Q}_{L}, \tilde{U}_{R}, \tilde{D}_{R}, \tilde{L}_{L}, \tilde{E}_{R}}, M_{\tilde{Q}_{3 L}, \tilde{t}_{R}, \tilde{b}_{R}, \tilde{L}_{3 L}, \tilde{\tau}_{R}}, \\
& M_{H^{ \pm}}, \mu, \tan \beta, A_{t, b, \tau}
\end{aligned}
$$

and the six phases $\Phi_{\alpha}$ discussed previously. We perform a scan of the pMSSM parameter space using the iterative geometric approach described in Sect. 2. We first generated about 40 million points, and then kept only points with a neutral Higgs boson with a mass in the range 121-129 GeV

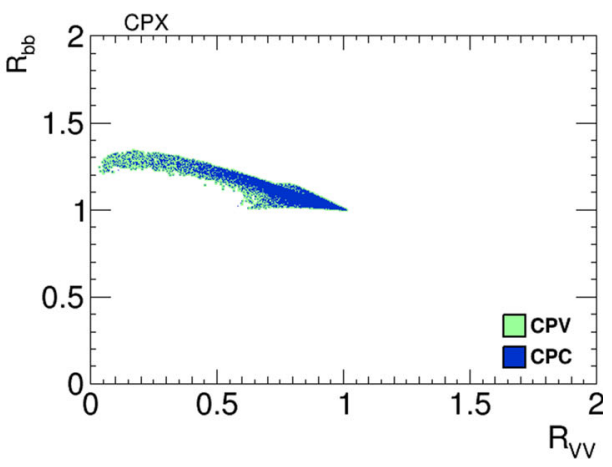

violating sample (green dots). The left panel displays a linear correlation between $R_{\gamma \gamma}$ and $R_{g g}$, and the right panel displays a nonlinear anti-correlation between $R_{V V}$ and $R_{\bar{b} b}$ 


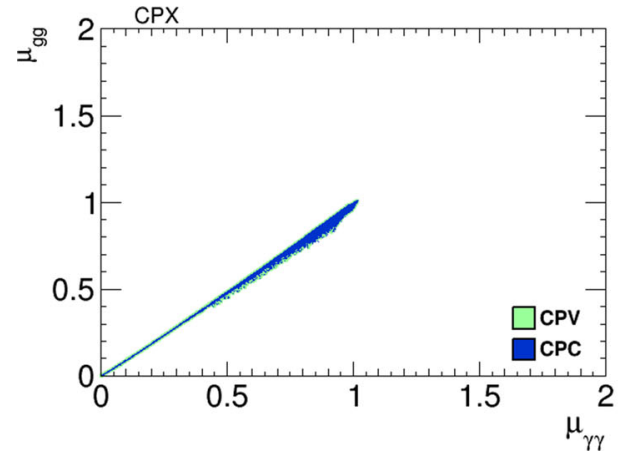

Fig. 19 Scatter plots of the $h_{1}$ signal strengths in the CPX scenario in the CP-violating limit $\Phi_{\alpha}=0$ (blue dots) and in the CP-violating sample (green dots). The left panel displays a strong linear correlation

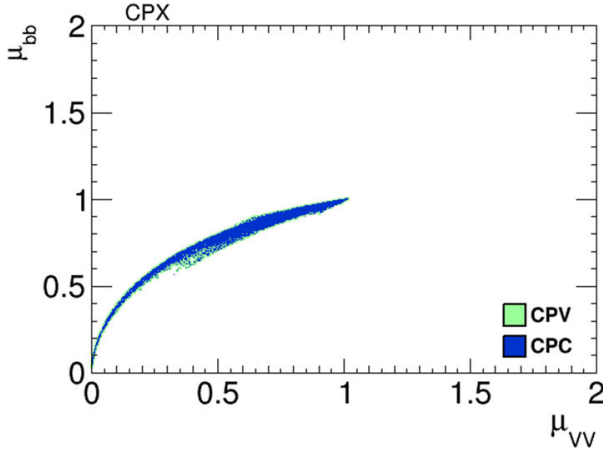

between $\mu_{\gamma \gamma}$ and $\mu_{g g}$, and the right panel displays a nonlinear correlation between $\mu_{V V}$ and $\mu_{\bar{b} b}$

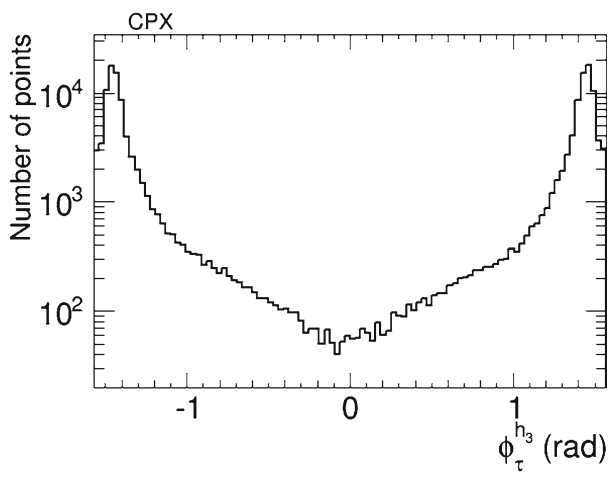

Fig. 20 The distributions of (left) the CP-violating phase $\phi_{\tau}^{h_{2}}$ in $h_{2} \tau \tau$ couplings and (right) the CP-violating phase $\phi_{\tau}^{h_{2}}$ in $h_{2} \tau \tau$ couplings in the CPX scenario $(9,10)$, found after applying all the constraints using the geometric approach described in the text
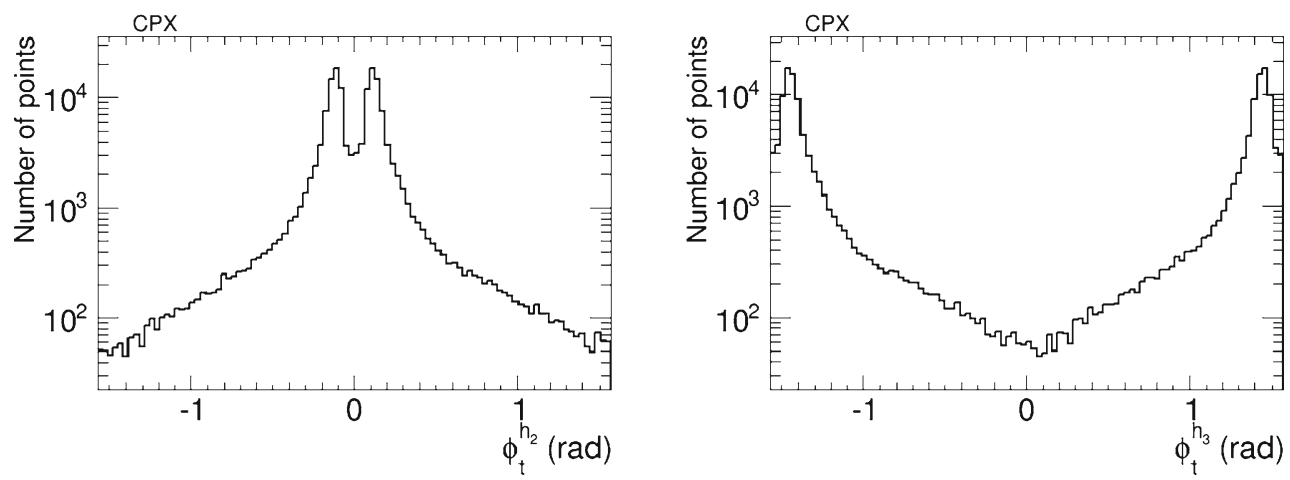

Fig. 21 The distributions of (left) the CP-violating phase $\phi_{t}^{h_{2}}$ in $h_{2} \bar{t} t$ couplings and (right) the CP-violating phase $\phi_{t}^{h_{2}}$ in $h_{2} \bar{t} t$ couplings in the CPX scenario $(9,10)$, found after applying all the constraints using the geometric approach described in the text

(thereby allowing for a conservative theoretical uncertainty in the Higgs mass calculation), and with a neutralino LSP. These requirements reduced the number of points to about 1 million. Imposing the EDM constraints then left about 150000 valid points. In the following plots, in addition to these constraints, we also impose flavour constraints, the cosmological upper bound on the dark matter density, the LUX direct upper limit on spin-independent dark matter scattering (except when the same observable is plotted), and we require squarks and the gluino to have masses above $500 \mathrm{GeV}$.
Figure 22 shows the samplings of the phases $\Phi_{\alpha}$ obtained after imposing these constraints. We see that values of $\Phi_{A_{t, b}}$ and $\Phi_{1} \sim \pm 90^{\circ}$ are quite well sampled, as are values of $\Phi_{A_{\tau}} \sim 90^{\circ}$. On the other hand, large values of $\Phi_{3}$ are less well sampled, and the range of $\Phi_{2}$ is very restricted with only small deviations from the $\mathrm{CP}$-conserving cases being allowed.

We see in Fig. 23 the extent to which the EDM constraints impose cancellations $\Phi_{3}$ and $\Phi_{A_{t}}$ (left panel) and between $\Phi_{3}$ and $\Phi_{A_{b}}$ (right panel). We see that large val- 

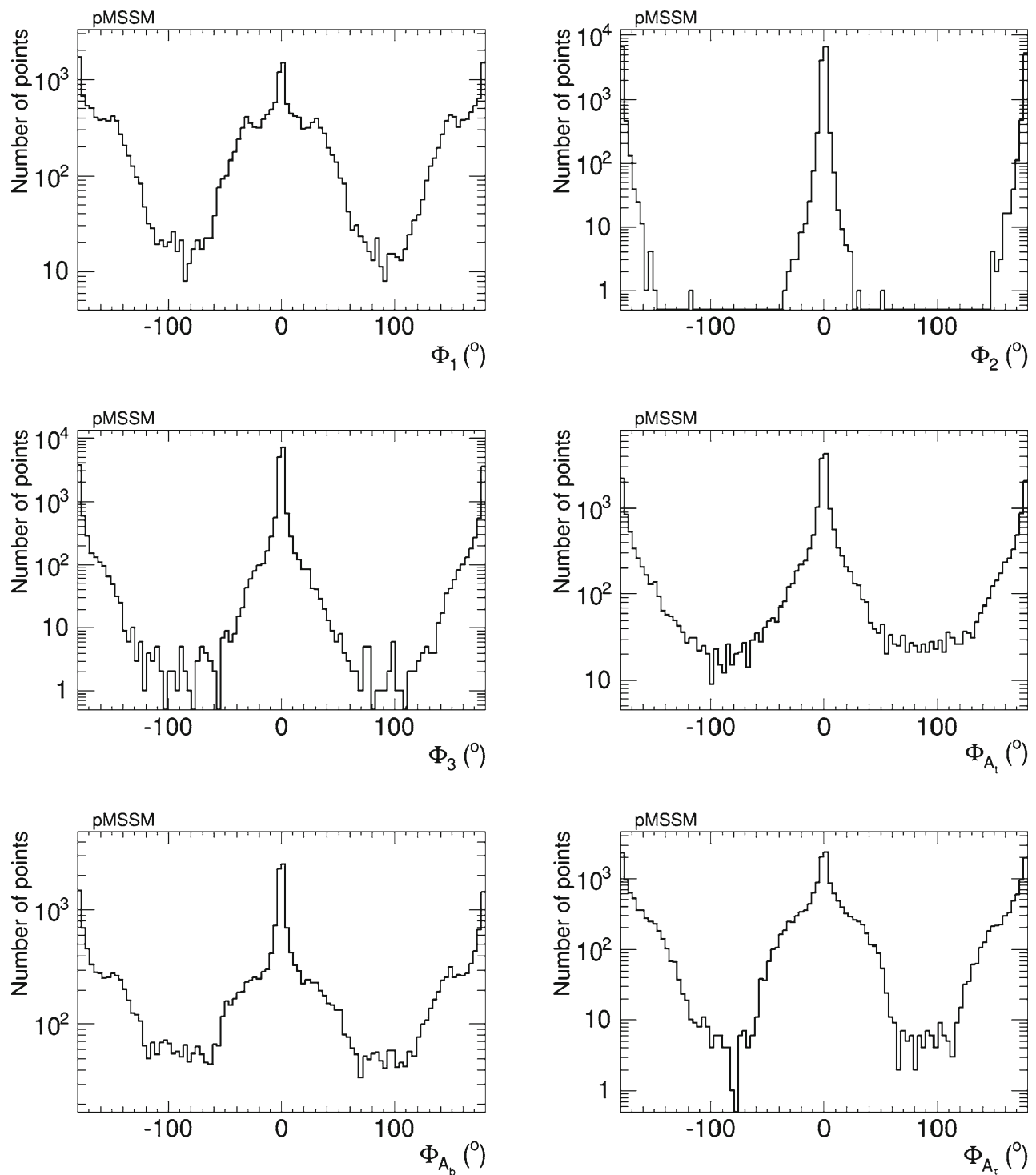

Fig. 22 Sampling of the CP-violating phases $\Phi_{\alpha}$ in the pMSSM scenario generated in the iterative geometric approach, imposing the EDM and other constraints
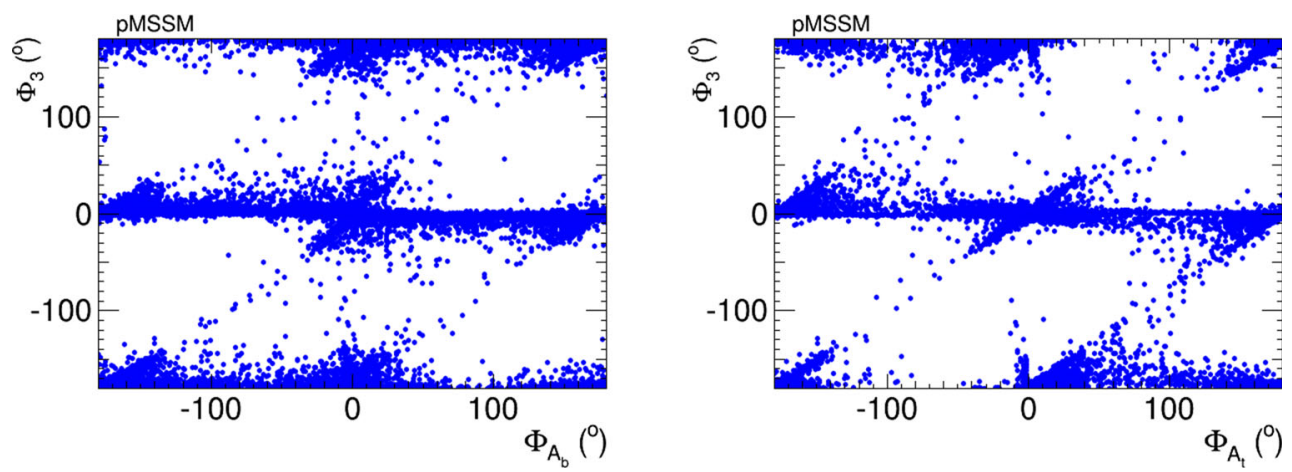

Fig. 23 Correlations of $\Phi_{3}$ with $\Phi_{\mathrm{A}_{\mathrm{b}}}$ (left panel) and $\Phi_{3}$ with $\Phi_{\mathrm{A}_{\mathrm{t}}}$ (right panel) imposed by the EDM constraints in the pMSSM scenario 


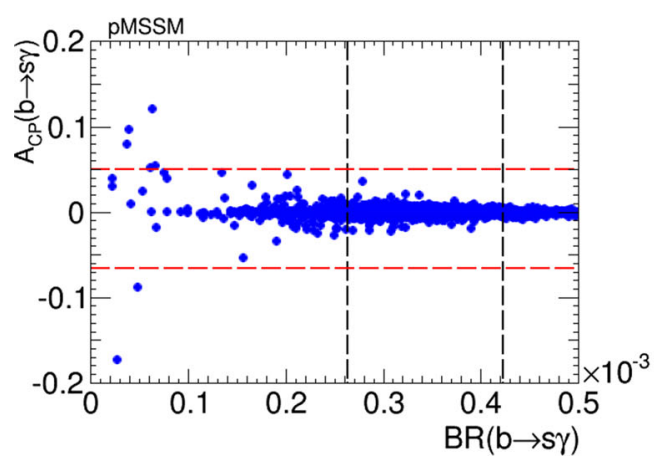

Fig. 24 Left panel Scatter plot of the branching ratio for $b \rightarrow s \gamma$ decay versus its $\mathrm{CP}$-violating asymmetry, $A_{\mathrm{CP}}$, in the pMSSM scenario. The vertical black dashed lines represent the allowed range for the $b \rightarrow s \gamma$ branching ratio, and the horizontal red dashed lines represent the present experimental limits on $A_{\mathrm{CP}}$. Right panel Histogram of

ues of $\left(\Phi_{A_{t}, A_{b}}, \Phi_{3}\right) \sim\left( \pm 90^{\circ}, \pm 90^{\circ}\right)$ are allowed, and we also see diagonal features corresponding to correlations. As in the NUHM2, it is apparent that the EDM constraints do not require all the $\mathrm{CP}$-violating phases to be small simultaneously.

The left panel of Fig. 24 displays a scatter plot of the values of $A_{\mathrm{CP}}$ found in the pMSSM using the iterative geometric approach. We see that values $\lesssim 3 \%$ are possible for values of the $b \rightarrow s \gamma$ branching ratio lying within the experimentally allowed range. The right panel of Fig. 24 shows a histogram of $A_{\mathrm{CP}}$ values, imposing only the Higgs mass and EDM cuts. Here we see tails extending to larger values of $\left|A_{\mathrm{CP}}\right|$ that lie outside the experimentally allowed range when the EDM constraints are not applied (grey histogram), whereas the black histogram is for points satisfying the EDM constraints. The vertical red dashed lines show the present experimental constraints on $A_{\mathrm{CP}}$, and the possible future improvement in the experimental sensitivity by a factor of 10 is indicated by vertical green dashed lines. As in the NUHM2, there are CPviolating pMSSM parameter sets that could be explored with such an improvement: it would provide additional information on $\mathrm{CP}$ violation within the pMSSM.

The possible new physics contribution to $B_{s}$ meson mass mixing, $\Delta M_{B_{s}}^{\mathrm{NP}}$, in the pMSSM scenario is shown in Fig. 25. As in the previous cases studied, the grey histogram is for the full sample, and the black histogram is for points that also satisfy the EDM constraints. If the theoretical uncertainty in the Standard Model contribution to $B_{S}$ mixing could be reduced by a factor of 10 thanks to improved lattice calculations, the sensitivity to $\Delta M_{B_{s}}^{\mathrm{NP}}$ would become that indicated by the vertical yellow dashed line in Fig. 25. In this case, many of the pMSSM models that are currently viable (indicated by the black histogram) could be explored.

In Fig. 26, we show in the left panel the values of the relic LSP density $\Omega_{\chi} h^{2}$ that we find in our pMSSM scan, and in right panel we show values of the spin-independent dark

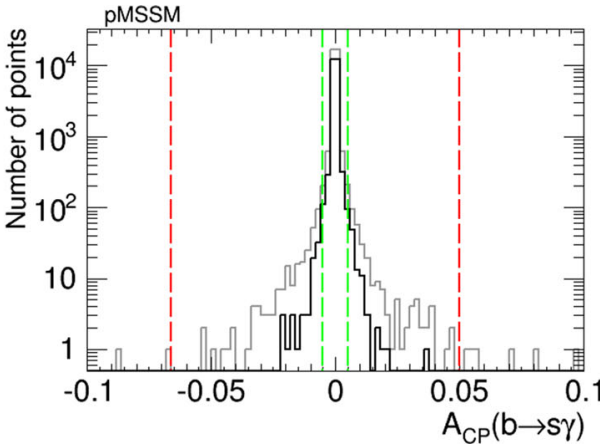

$A_{\mathrm{CP}}$ in the pMSSM, imposing only the Higgs mass and EDM cuts (grey full sample, black points satisfying the EDM constraints). The vertical red dashed lines represent the present experimental limits, and the vertical green dashed lines represent the prospective future improvement in the sensitivity to $A_{\mathrm{CP}}$ by a factor of 10

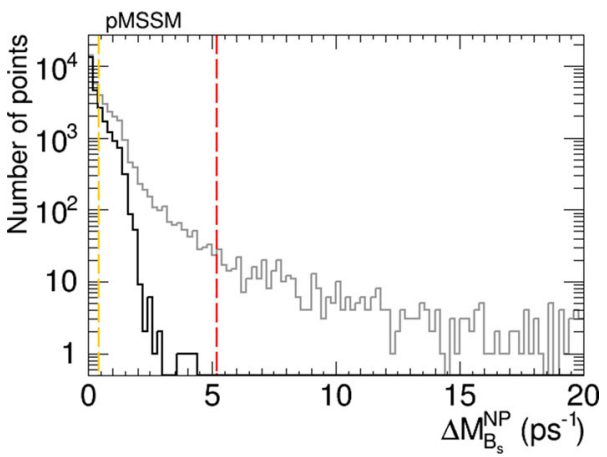

Fig. 25 Histogram of the possible new physics contribution to $B_{S}$ mixing, $\Delta M_{B_{s}}^{\mathrm{NP}}$, in the pMSSM scenario. The grey histogram is for points satisfying the Higgs mass and other constraints, and the black histogram is for points that also satisfy the EDM constraints. The vertical red dashed line is the present experimental upper limit on $\Delta M_{B_{s}}^{\mathrm{NP}}$, and the vertical yellow dashed line shows the potential of a reduction in the current theoretical uncertainty in the Standard Model by a factor of 10

matter scattering cross section $\sigma_{\mathrm{SI}}^{p} v$. We see that values of $\Omega_{\chi} h^{2}$ considerably above the cosmological upper limit are possible in both the CP-conserving (blue dots) and the CPviolating cases (green dots). We also see in the right panel of Fig. 26 that values of $\sigma_{\mathrm{SI}}^{p} v$ above the LUX upper limit are also possible. In both panels, there are no large differences between the $\mathrm{CP}$-conserving and $\mathrm{CP}$-violating cases.

Scatter plots of values of $h_{1}$ branching ratios in the pMSSM scenario are in Fig. 27, the left panel displaying $\left(R_{\gamma \gamma}, R_{g g}\right)$ and the right panel displaying $\left(R_{V V}, R_{\bar{b} b}\right)$. As previously, the blue dots are CP-conserving parameter choices with $\Phi_{\alpha}=0$, and the green dots are from a scan of CP-violating points with $\Phi_{\alpha} \neq 0$.

As in the NUHM2 scenario, we note in the left panel a strong correlation between $R_{\gamma \gamma}$ and $R_{g g}$, which may be either much smaller than in the Standard Model or somewhat larger, and we also see in the right panel that a large reduction in $R_{V V}$ is possible. Also as in the NUHM2 scenario, the reduc- 


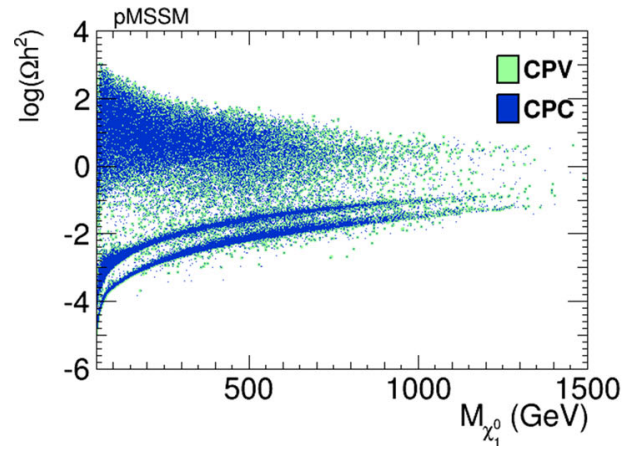

Fig. 26 Left panel Scatter plot of the dark matter relic density as a function of the neutralino mass in the pMSSM scenario. Right panel Scatter plot of the spin-independent dark matter scattering cross sec-

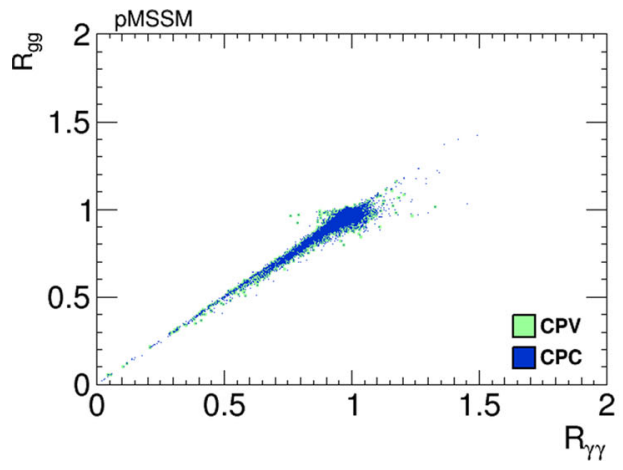

Fig. 27 Scatter plots of branching ratios, normalised to the Standard Model values, for decays of the lightest Higgs boson, $h_{1}$, in the pMSSM scenario in the CP-violating limit $\Phi_{\alpha}=0$ (blue dots) and in the CP-

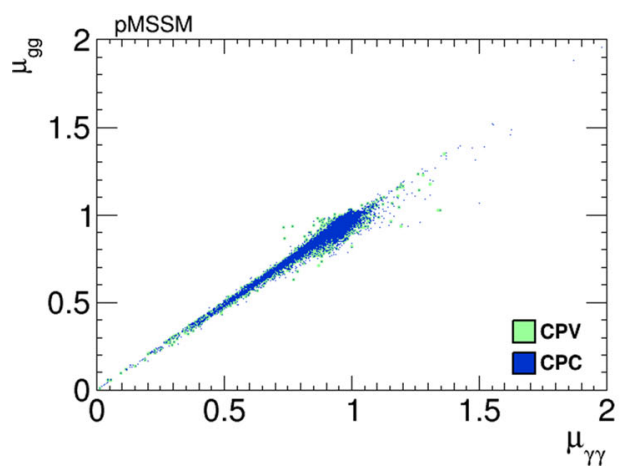

Fig. 28 Scatter plots of the $h_{1}$ signal strengths in the pMSSM scenario in the CP-violating limit $\Phi_{\alpha}=0$ (blue dots) and in the CP-violating sample (green dots). The left panel displays a strong linear correlation

tion in $R_{V V}$ may be accompanied by values of $R_{\bar{b} b}$ that are either larger or smaller than in the Standard Model, the latter possibility arising when the Higgs boson can decay into light sparticles.

Figure 28 displays scatter plots of $h_{1}$ signal strengths $\mu_{X}$ in the pMSSM scenario in the CP-conserving case with phases $\Phi_{\alpha}=0$ (blue dots) and in the CP-violating case where the $\Phi_{\alpha} \neq 0$ (green dots). As in the NUHM2 case, we see a strong correlation between $\mu_{\gamma \gamma}$ and $\mu_{g g}$ in the left panel, and

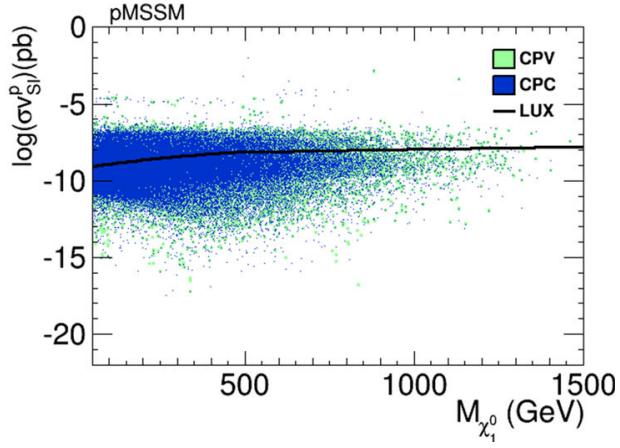

tion $\sigma_{\mathrm{SI}}^{p} v$ as a function of the neutralino mass in the pMSSM scenario. In both panels, $\mathrm{CP}$-conserving parameter choices are denoted by blue dots, and CP-violating parameter choices by green dots

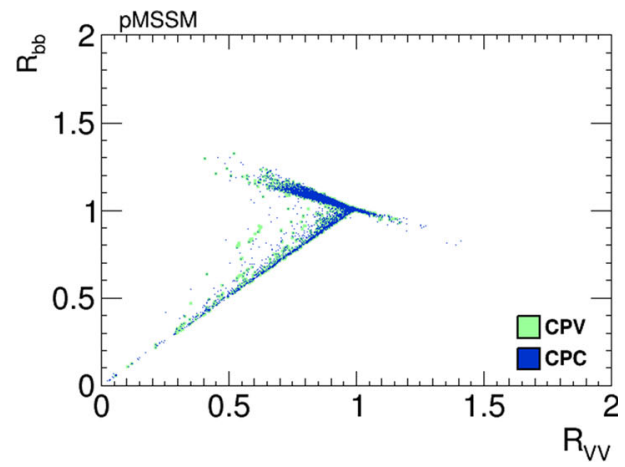

violating sample (green dots). The left panel displays a strong correlation between $R_{\gamma \gamma}$ and $R_{g g}$, and the right panel displays a bimodal correlation between $R_{V V}$ and $R_{\bar{b} b}$

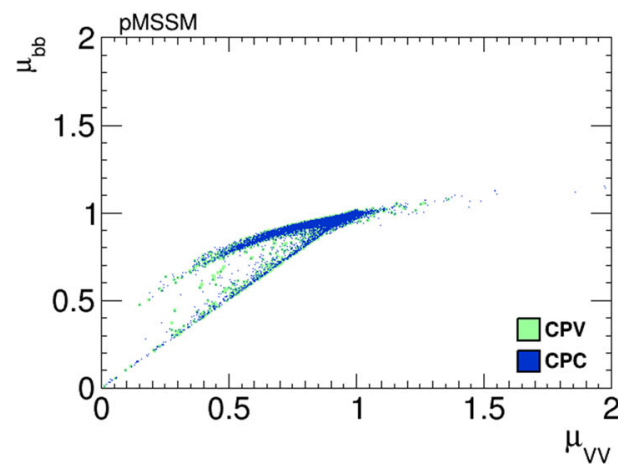

between $\mu_{\gamma \gamma}$ and $\mu_{g g}$, and the right panel displays a bimodal correlation between $\mu_{V V}$ and $\mu_{\bar{b} b}$ for smaller values

in the right panel we see a correlation between $\mu_{V V}$ and $\mu_{\bar{b} b}$ that becomes bimodal for small values of $\mu_{V V}$.

We have also studied whether the Higgs boson discovered at the LHC might be one of the heavier Higgs bosons in the pMSSM, with or without CP violation. As seen in the left panel of Fig. 29, if the known Higgs boson is identified with the $h_{2}$, it is not possible to satisfy the Higgs signal strength constraints. This is possible if the discovered Higgs boson is identified with the $h_{3}$, as seen (green dots) in the right panel 


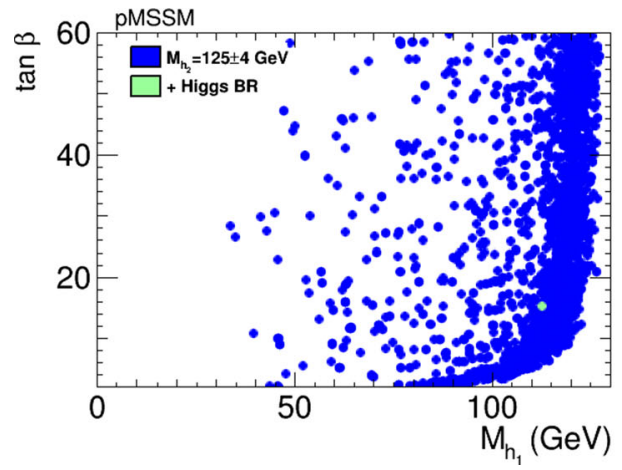

Fig. 29 Scatter plots of pMSSM points in the $\left(m_{h_{1}}, \tan \beta\right)$ plane in the case where either the $h_{2}$ (left panel) or the $h_{3}$ (right panel) is the Higgs boson discovered at the LHC, applying only the EDM and Higgs

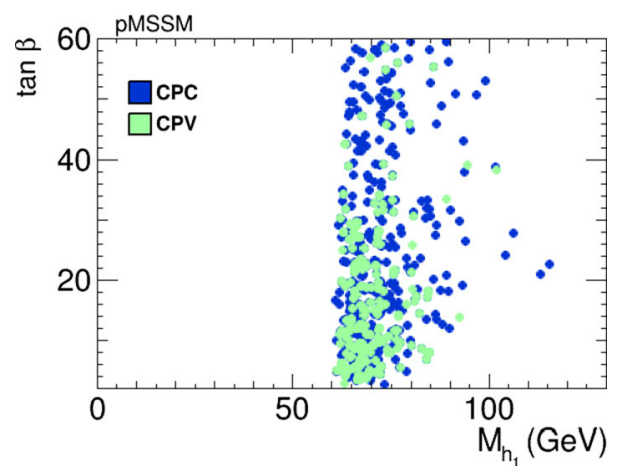

Fig. 30 Scatter plot of pMSSM points in the $\left(m_{h_{1}}, \tan \beta\right)$ plane in the case where the Higgs boson discovered at the LHC is identified as the $h_{3}$, for the points satisfying the Higgs signal strength constraints as well as the EDM constraints

of Fig. 29, in which case the $h_{1}$ mass is about $60-80 \mathrm{GeV}$. Figure 30 displays these points in both the $\mathrm{CP}$-conserving case (blue dots) and the CP-violating case (green dots), which are quite similar. On the other hand, none of these points survive the charged Higgs and $A / H \rightarrow \tau \tau$ constraints, nor the flavour constraints. We therefore conclude that the pMSSM does not provide a way to conceal a neutral Higgs boson that is lighter than the one discovered, even if CP is violated.

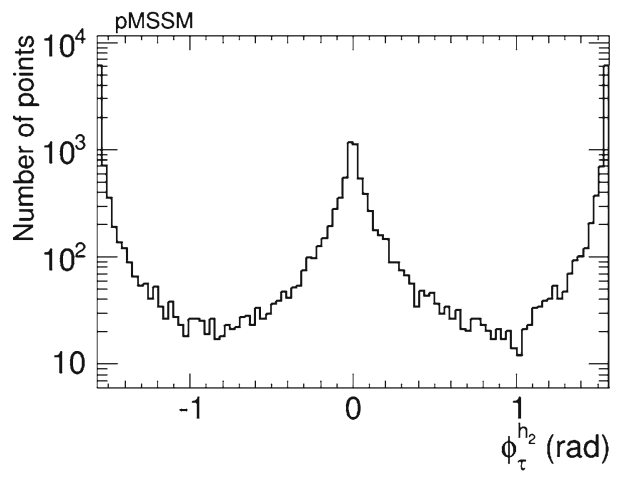

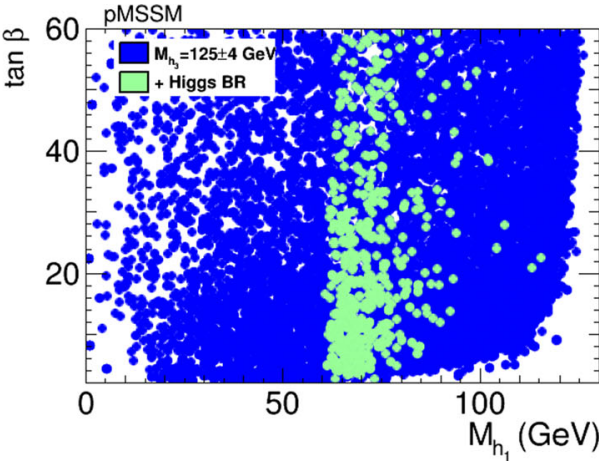

mass constraints (blue dots), and applying also the Higgs signal strength constraints (green dots). We find no points that satisfy in addition the neutral and charged heavy Higgs search constraints

Assuming that the Higgs boson discovered at the LHC is indeed the lightest MSSM Higgs boson $h_{1}$, we now assess the prospects for $\mathrm{CP}$ violation in the couplings of the heavy neutral Higgs bosons to $\tau^{+} \tau^{-}$and $\bar{t} t$ in the pMSSM scenario (11) which are shown in Figs. 31 and 32. We see that, as in the CMSSM, CPX and NUHM2 cases discussed previously, $h_{2,3}$ decays may provide interesting prospects for probing $\mathrm{CP}$ violation also in this pMSSM scenario. On the other hand, we again find that after imposing all the constraints the phases for the $h_{1}$ couplings are small, namely $\phi_{\tau}^{h_{1}} \lesssim 0.03$ radians and $\phi_{t}^{h_{1}} \lesssim 0.02$ radians, respectively.

\section{Conclusions}

The geometrical approach to implementing EDM constraints and maximizing other $\mathrm{CP}$-violating observables proposed in [2] provides a suitable way to explore the possibilities for $\mathrm{CP}$ violation in variants of the MSSM, which we have applied in this paper to explore the CMSSM, the CPX scenario, the NUHM2 and the pMSSM. We have adopted an iterative extension of the geometric approach, which is suitable for exploring larger values of the $\mathrm{CP}$-violating phases. Our

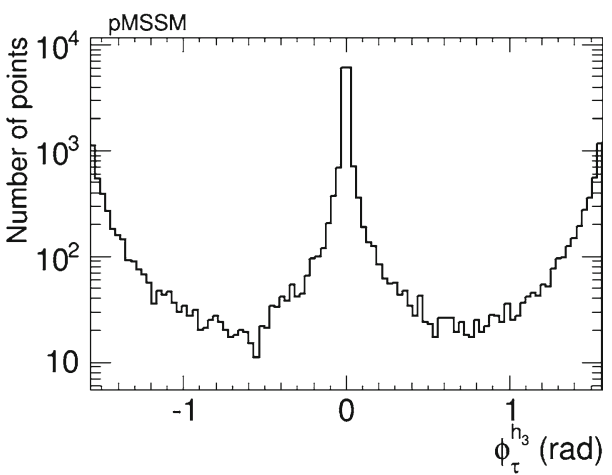

Fig. 31 The distributions of (left) the CP-violating phase $\phi_{\tau}^{h_{2}}$ in $h_{2} \tau \tau$ couplings and (right) the CP-violating phase $\phi_{\tau}^{h_{3}}$ in $h_{3} \tau \tau$ couplings in the pMSSM scenario (11), found after applying all the constraints using the geometric approach described in the text 

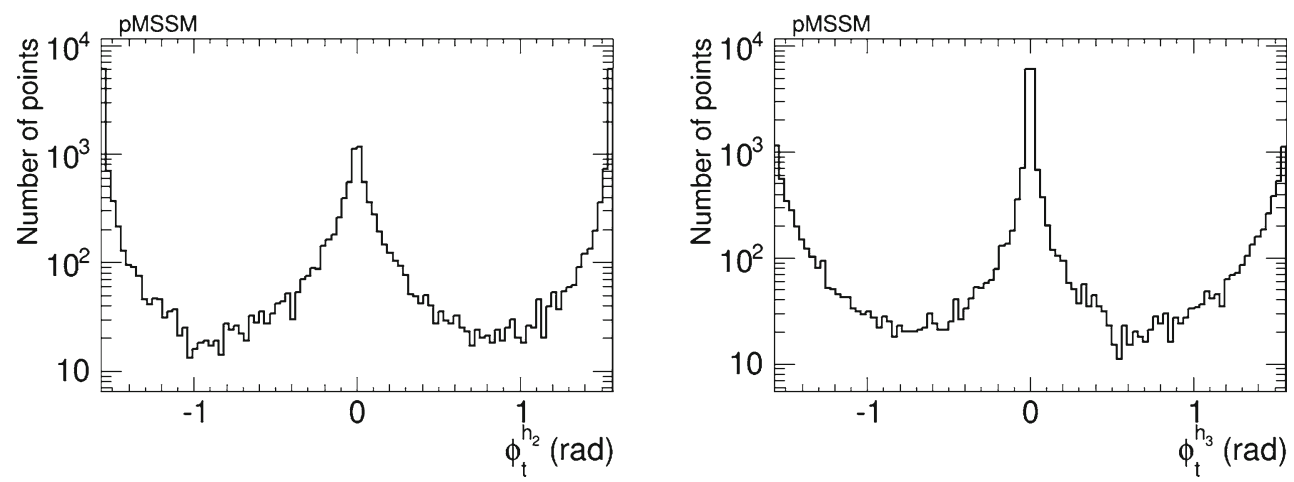

Fig. 32 The distributions of (left) the CP-violating phase $\phi_{t}^{h_{2}}$ in $h_{2} \bar{t} t$ couplings and (right) the CP-violating phase $\phi_{t}^{h_{3}}$ in $h_{3} \bar{t} t$ couplings in the pMSSM scenario (11), found after applying all the constraints using the geometric approach described in the text

explorations have been within the maximally $\mathrm{CP}$-violating, minimal flavour-violating (MCPMFV) framework with six $\mathrm{CP}$-violating phases, of which two combinations are unconstrained a priori by the four EDM constraints. The following are our principal results:

- In the CMSSM we have explored CP-violating generalisations of the low-mass best-fit point (6) that was identified in [11,12], where we found relatively little scope for large deviations from the $\mathrm{CP}$-conserving case, e.g., in the masses of the Higgs bosons and the spin-independent dark matter scattering cross section. Moreover, we found that only very small values of $A_{\mathrm{CP}} \lesssim 0.001$ would be possible in this case, and the new physics contribution to $B_{s}$ meson mixing, $\Delta M_{B_{s}}^{\mathrm{NP}}$, would not be observable.

- We have then explored the CPX scenario (9), where we also found no scope for measurable values of $A_{\mathrm{CP}}$. On the other hand, we found in this model that $\Delta M_{B_{s}}^{\mathrm{NP}}$ could be large enough to provide a possible signature if the current lattice theoretical uncertainty in the Standard Model contribution to $B_{S}$ mixing could be reduced by a factor of 10, as seen in Fig. 17.

- The situation in the NUHM2 scenario (8) is rather more favourable for observable signals of $\mathrm{CP}$ violation. In this case, $A_{\mathrm{CP}}$ could be as large as $\sim 2 \%$ and hence lie well within the reach of experiment, as seen in Fig. 9, and $\Delta M_{B_{s}}^{\mathrm{NP}}$ might also be large enough to provide a possible experimental signature, as seen in Fig. 10.

- A similar situation was found in the pMSSM scenario (11), in which case $A_{\mathrm{CP}}$ could be as large as $\sim 3 \%$, as seen in Fig. 24, again within the reach of experiment. We also find in this scenario that $\Delta M_{B_{s}}^{\mathrm{NP}}$ could be large enough to be observable with a prospective reduction in the theoretical uncertainty in the Standard Model calculation of $B_{s}$ mixing, as seen in Fig. 25.

- In all the scenarios studied, the CP-violating phases in the $h_{1} \tau^{+} \tau^{-}$and $h_{1} \bar{t} t$ couplings are small. On the other hand, the phases in the $h_{2,3} \tau^{+} \tau^{-}$and $h_{2,3} \bar{t} t$ couplings can be quite large, and may present interesting prospects for future $p p, e^{+} e^{-}$and $\mu^{+} \mu^{-}$experiments, though their detailed study lies beyond the scope of this work.

Our analysis serves as a reminder that the EDM constraints do not force all the six non-KM CP-violating phases in MCPMFV to be small, and that in some variants of the MSSM there could be observable signatures of $\mathrm{CP}$ violation beyond the Standard Model, e.g., $A_{\mathrm{CP}}$ in $b \rightarrow s \gamma$ decay. We look forward to a generation of $A_{\mathrm{CP}}$ measurements, and also to improved theoretical calculations of the Standard Model contribution to $B_{s}$ meson mixing, which might enable a new physics contribution $\Delta M_{B_{s}}^{\mathrm{NP}}$ to be isolated. If enough soft supersymmetry-breaking parameters could be measured, and both $A_{\mathrm{CP}}$ and $\Delta M_{B_{s}}^{\mathrm{NP}}$ could be shown to have measurable deviations from the Standard Model, one might finally be able to fix all the six non-KM CP-violating phases in MCPMFV.

Acknowledgments The work of J.E. was supported in part by the London Centre for Terauniverse Studies (LCTS), using funding from the European Research Council via the Advanced Investigator Grant 267352 and from the UK STFC via the research Grant ST/J002798/1. The work of A.A. was supported in part by the Fédération de Recherche A.-M. Ampère de Lyon. R.M.G. wishes to acknowledge support from the Department of Science and Technology, India under Grant No. SR/S2/JCB-64/2007 under the J.C. Bose Fellowship scheme and hospitality in the CERN theory division.

Open Access This article is distributed under the terms of the Creative Commons Attribution License which permits any use, distribution, and reproduction in any medium, provided the original author(s) and the source are credited.

Funded by $\mathrm{SCOAP}^{3}$ / License Version CC BY 4.0.

\section{References}

1. J.R. Ellis, J.S. Lee, A. Pilaftsis, B-Meson observables in the maximally CP-violating MSSM with minimal flavour violation. Phys. Rev. D 76, 115011 (2007). arXiv:0708.2079 [hep-ph] 
2. J. Ellis, J.S. Lee, A. Pilaftsis, A geometric approach to CP violation: applications to the MCPMFV SUSY model. JHEP 1010, 049 (2010). arXiv:1006.3087 [hep-ph]

3. T. Gajdosik, R.M. Godbole, S. Kraml, Fermion polarization in sfermion decays as a probe of CP phases in the MSSM. JHEP 0409, 051 (2004). arXiv:hep-ph/0405167

4. K.A. Olive, M. Pospelov, A. Ritz, Y. Santoso, CP-odd phase correlations and electric dipole moments. Phys. Rev. D 72, 075001 (2005). arXiv:hep-ph/0506106

5. S.A.R. Ellis, G.L. Kane, Theoretical prediction and impact of fundamental electric dipole moments. arXiv:1405.7719 [hep-ph]

6. B.C. Regan, E.D. Commins, C.J. Schmidt, D. DeMille, New limit on the electron electric dipole moment. Phys. Rev. Lett. 88, 071805 (2002)

7. W.C. Griffith, M.D. Swallows, T.H. Loftus, M.V. Romalis, B.R. Heckel, E.N. Fortson, Improved limit on the permanent electric dipole moment of Hg-199. Phys. Rev. Lett. 102, 101601 (2009)

8. C.A. Baker, D.D. Doyle, P. Geltenbort, K. Green, M.G.D. van der Grinten, P.G. Harris, P. Iaydjiev, S.N. Ivanov et al., An Improved experimental limit on the electric dipole moment of the neutron. Phys. Rev. Lett. 97, 131801 (2006). arXiv:hep-ex/0602020

9. J. Baron et al. (ACME Collaboration), Order of magnitude smaller limit on the electric dipole moment of the electron. Science 343(6168), 269 (2014). arXiv:1310.7534 [physics.atom-ph]

10. G.W. Bennett et al. (Muon (g-2) Collaboration), An improved limit on the muon electric dipole moment. Phys. Rev. D 80, 052008 (2009). arXiv:0811.1207 [hep-ex]

11. O. Buchmueller, R. Cavanaugh, A. De Roeck, M.J. Dolan, J.R. Ellis, H. Flacher, S. Heinemeyer, G. Isidori et al., The CMSSM and NUHM1 after LHC run 1. Eur. Phys. J. C 74, 2922 (2014). arXiv: 1312.5250 [hep-ph]

12. O. Buchmueller, R. Cavanaugh, M. Citron, A. De Roeck, M.J. Dolan, J.R. Ellis, H. Flaecher, S. Heinemeyer et al., The NUHM2 after LHC run 1. arXiv:1408.4060 [hep-ph]

13. M.S. Carena, J.R. Ellis, A. Pilaftsis, C.E.M. Wagner, CP violating MSSM Higgs bosons in the light of LEP-2. Phys. Lett. B 495, 155 (2000). arXiv:hep-ph/0009212

14. A. Djouadi et al. (MSSM Working Group Collaboration), The minimal supersymmetric standard model: group summary report. arXiv:hep-ph/9901246

15. G. Brooijmans et al., Les Houches 2013: physics at TeV colliders: new physics working group report. arXiv:1405.1617 [hep-ph]

16. A. Arbey, M. Battaglia, F. Mahmoudi, Constraints on the MSSM from the Higgs sector: a pMSSM study of Higgs searches, $B_{s}^{0} \rightarrow$ $\mu^{+} \mu^{-}$and dark matter direct detection. Eur. Phys. J. C 72, 1906 (2012). arXiv:1112.3032 [hep-ph]

17. A. Arbey, M. Battaglia, F. Mahmoudi, Complementarity of WIMP sensitivity with direct SUSY, monojet and dark matter searches in the MSSM. Phys. Rev. D 89, 077701 (2014). arXiv:1311.7641 [hep-ph]

18. M. Cahill-Rowley, J.L. Hewett, A. Ismail, T.G. Rizzo, Lessons and prospects from the pMSSM after LHC run I: neutralino LSP. arXiv: 1407.4130 [hep-ph]

19. J.S. Lee, A. Pilaftsis, M.S. Carena, S.Y. Choi, M. Drees, J.R. Ellis, C.E.M. Wagner, CPsuperH: a computational tool for Higgs phenomenology in the minimal supersymmetric standard model with explicit CP violation. Comput. Phys. Commun. 156, 283 (2004). arXiv:hep-ph/0307377

20. J.S. Lee, M. Carena, J. Ellis, A. Pilaftsis, C.E.M. Wagner, CPsuperH2.0: an improved computational tool for Higgs phenomenology in the MSSM with explicit CP violation. Comput. Phys. Commun. 180, 312 (2009). arXiv:0712.2360 [hep-ph]

21. J.S. Lee, M. Carena, J. Ellis, A. Pilaftsis, C.E.M. Wagner, CPsuperH2.3: an updated tool for phenomenology in the MSSM with explicit CP violation. Comput. Phys. Commun. 184, 1220 (2013). arXiv:1208.2212 [hep-ph]

22. K. Cheung, J.S. Lee, E. Senaha, P.Y. Tseng, Confronting Higgcision with electric dipole moments. JHEP 1406, 149 (2014). arXiv:1403.4775 [hep-ph]

23. F. Mahmoudi, SuperIso: a program for calculating the isospin asymmetry of $B \rightarrow K^{*} \gamma$ in the MSSM. Comput. Phys. Commun. 178, 745 (2008). arXiv:0710.2067 [hep-ph]

24. F. Mahmoudi, SuperIso v2.3: a program for calculating flavor physics observables in supersymmetry. Comput. Phys. Commun. 180, 1579 (2009). arXiv:0808.3144 [hep-ph]

25. A. Arbey, F. Mahmoudi, SuperIso relic: a program for calculating relic density and flavor physics observables in supersymmetry. Comput. Phys. Commun. 181, 1277 (2010). arXiv:0906.0369 [hep$\mathrm{ph}]$

26. G. Belanger, F. Boudjema, A. Pukhov, A. Semenov, MicrOMEGAs 2.0: a program to calculate the relic density of dark matter in a generic model. Comput. Phys. Commun. 176, 367 (2007). arXiv:hep-ph/0607059

27. G. Belanger, F. Boudjema, A. Pukhov, A. Semenov, Dark matter direct detection rate in a generic model with micrOMEGAs 2.2 Comput. Phys. Commun. 180, 747 (2009). arXiv:0803.2360 [hep$\mathrm{ph}]$

28. G. Belanger, F. Boudjema, A. Pukhov, A. Semenov, micrOMEGAs 3: a program for calculating dark matter observables. Comput. Phys. Commun. 185, 960 (2014). arXiv: 1305.0237 [hep-ph]

29. P. Bechtle, O. Brein, S. Heinemeyer, O. Stål, T. Stefaniak, G. Weiglein, K.E. Williams, HiggsBounds - 4: improved tests of extended Higgs sectors against exclusion bounds from LEP, the Tevatron and the LHC. Eur. Phys. J. C 74, 2693 (2014). arXiv: 1311.0055 [hep-ph]

30. A. Lenz, U. Nierste, Numerical updates of lifetimes and mixing parameters of B mesons. arXiv:1102.4274 [hep-ph]

31. See for example, J. Ellis, D.S. Hwang, K. Sakurai, M. Takeuchi, JHEP 1404, 004 (2014). arXiv:1312.5736 [hep-ph]

32. F. Boudjema, R.M. Godbole, D. Guadagnoli, K. Mohan, in Ref. [15]

33. See for example, J.R. Ellis, J.S. Lee, A. Pilaftsis, Nucl. Phys. B 718, 247 (2005). arXiv:hep-ph/0411379

34. S.Y. Choi, J. Kalinowski, Y. Liao, P.M. Zerwas, Eur. Phys. J. C 40, 555 (2005). arXiv:hep-ph/0407347

35. J.R. Ellis, J.S. Lee, A. Pilaftsis, Phys. Rev. D 72, 095006 (2005). arXiv:hep-ph/0507046

36. R.M. Godbole, S. Kraml, S.D. Rindani, R.K. Singh, Phys. Rev. D 74, 095006 (2006). arXiv:hep-ph/0609113 [Erratum-ibid. D 74, 119901 (2006)]

37. J.R. Ellis, J.S. Lee, A. Pilaftsis, CERN LHC signatures of resonant CP violation in a minimal supersymmetric Higgs sector. Phys. Rev. D 70, 075010 (2004). arXiv:hep-ph/0404167

38. S. Berge, W. Bernreuther, B. Niepelt, H. Spiesberger, How to pin down the CP quantum numbers of a Higgs boson in its tau decays at the LHC. Phys. Rev. D 84, 116003 (2011). arXiv:1108.0670 [hep-ph]

39. A. Chakraborty, B. Das, J.L. Diaz-Cruz, D.K. Ghosh, S. Moretti, P. Poulose, The $125 \mathrm{GeV}$ Higgs signal at the LHC in the CP violating MSSM. Phys. Rev. D 90, 055005 (2014). arXiv:1301.2745 [hep$\mathrm{ph}]$

40. K.A. Olive et al. (Particle Data Group Collaboration), Review of particle physics. Chin. Phys. C 38, 090001 (2014)

41. T. Aushev, W. Bartel, A. Bondar, J. Brodzicka, T.E. Browder, P. Chang, Y. Chao, K.F. Chen et al., Physics at super B factory. arXiv: 1002.5012 [hep-ex]

42. D.S. Akerib et al. (LUX Collaboration), First results from the LUX dark matter experiment at the Sanford Underground Research 
Facility. Phys. Rev. Lett. 112, 091303 (2014). arXiv:1310.8214 [astro-ph.CO]

43. D. Albornoz Vasquez, G. Belanger, R.M. Godbole, A. Pukhov, The Higgs boson in the MSSM in light of the LHC. Phys. Rev. D 85, 115013 (2012). arXiv:1112.2200 [hep-ph]

44. A. Arbey, M. Battaglia, A. Djouadi, F. Mahmoudi, The Higgs sector of the phenomenological MSSM in the light of the Higgs boson discovery. JHEP 1209, 107 (2012). arXiv:1207.1348 [hep-ph]
45. A. Arbey, M. Battaglia, A. Djouadi, F. Mahmoudi, An update on the constraints on the phenomenological MSSM from the new LHC Higgs results. Phys. Lett. B 720, 153 (2013). arXiv:1211.4004 [hep$\mathrm{ph}]$ 